\title{
Review on the phytochemistry and toxicological profiles of Aloe vera and Aloe ferox
}

Florence Nalimu $u^{1,2^{*}}$ (D) Joseph Oloro ${ }^{1,3}$, Ivan Kahwa ${ }^{1,4}$ and Patrick Engeu Ogwang ${ }^{1,4}$

\begin{abstract}
Background: Aloe vera and Aloe ferox have over the years been among the most sought-after Aloe species in the treatment of ailments worldwide. This review provides categorized literature on the phytochemical and scientifically proven toxicological profiles of $A$. vera and A. ferox to facilitate their exploitation in therapy.

Main body of the abstract: Original full-text research articles were searched in PubMed, ScienceDirect, Research gate, Google Scholar, and Wiley Online Library using specific phrases. Phenolic acids, flavonoids, tannins, and anthraquinones were the main phytochemical classes present in all the two Aloe species. Most of the phytochemical investigations and toxicity studies have been done on the leaves. Aloe vera and Aloe ferox contain unique phytoconstituents including anthraquinones, flavonoids, tannins, sterols, alkaloids, and volatile oils. Aloe vera hydroalcoholic leaf extract showed a toxic effect on Kabir chicks at the highest doses. The methanolic, aqueous, and supercritical carbon dioxide extracts of $A$. vera leaf gel were associated with no toxic effects. The aqueous leaf extract of $A$. ferox is well tolerated for short-term management of ailments but long-term administration may be associated with organ toxicity. Long-term administration of the preparations from $A$. vera leaves and roots was associated with toxic effects.
\end{abstract}

Short conclusion: This review provides beneficial information about the phytochemistry and toxicity of $A$. vera and A. ferox and their potential in the treatment of COVID-19 which up to date has no definite cure. Clinical trials need to be carried out to clearly understand the toxic effects of these species.

Keywords: Aloe vera, Aloe ferox, Aloe, Phytochemistry, Toxicity, Review, Safety

\section{Background}

Aloe species (family Asphodelaceae) are among the most widely used plants over centuries for treating various ailments, for esthetic, and skincare [1]. The Aloe genus comprises over 430 species including $A$. vera and $A$. ferox among others [2]. These species have been reported to have pharmacological activities including antiinflammatory, immunomodulatory, antibacterial,

\footnotetext{
* Correspondence: fnalimu@must.ac.ug

'Pharm-Bio Technology and Traditional Medicine Centre of Excellence, Mbarara University of Science and Technology, Mbarara, Uganda ${ }^{2}$ Department of Pharmaceutical Sciences, Faculty of Medicine, Mbarara University of Science and Technology, P.O. Box 1410, Mbarara, Uganda Full list of author information is available at the end of the article
}

antifungal, antiviral, antiproliferative, antidiabetic, laxative, wound healing, moisturizing, anti-aging, and skin protection [3-5].

Aloe species are increasingly being incorporated into different cosmetic products, health drinks, foods, and beverages due to the abovementioned beneficial biological activities of the phytochemicals found mainly in the leaves.

These phytochemicals include polysaccharides, flavonoids, carbohydrates, coumarins, tannins, chromones, alkaloids, anthraquinones, organic compounds, pyrones, phytosterols, anthrones, sterols, vitamins, proteins, and mineral constituents $[2,5,6]$. The variation in concentration of these chemical constituents is based on the 
plant part used, extraction process, solvent, stage of growth, and plant source.

Though beneficial, some of these phytochemicals may be associated with toxic effects [7]. Many researchers have established potential toxicities as well as risks associated with some plants and vegetables particularly hepatotoxicity, nephrotoxicity, and cancer $[8,9]$. Due to these risks, toxicological evaluation of medicinal plants has become one of the main concerns to assure their safe use $[10,11]$.

This review focuses on the phytochemistry and toxicology of $A$. vera and $A$. ferox, the two commercially popular species of Aloe. The present study will help in the standardization and quantification of the phytochemicals present in the Aloe species. It will also create awareness to the locals of the toxic effects that may be associated with the use of these species as medicine and future studies in humans.

\section{Main text}

The search was made in the databases of PubMed, ScienceDirect, Research gate, Google Scholar, and Wiley Online Library using the phrases "Genus Aloe," "A. vera, " "toxicology of Aloe species," "acute and subacute toxicity of Aloe species," safety, "A. ferox," and "phytochemistry of Aloe species." Published original full-text articles in English language on phytochemistry and toxicity of the Aloe species were retrieved.

\section{Phytochemistry of the Aloe species}

Aloe vera and Aloe ferox contain vast phytochemical classes including anthraquinones, chromones, anthrones, phenolic compounds, flavonoids, tannins, steroids, and alkaloids which contribute to their different pharmacological activities. The structures of the individual compounds are included (Figs. 1, 2, 3, 4, 5, 6, 7, 8, 9, 10, 11, $12,13,14,15,16,17,18,19$, and 20). More information on phytochemistry is summarized in Tables 1,2 , and 3 .

\section{Acute toxicity}

According to Celestino et al. [51], A. ferox resin at a dose of $5000 \mathrm{mg} / \mathrm{kg}$ caused moderate diarrhea and reduced motor activity after $1 \mathrm{~h}$ post administration in Wistar rats.

Studies on both the methanolic and supercritical carbon dioxide extracts of $A$. vera leaf gel showed no treatment-related mortalities or changes in all the investigated parameters in rats $[56,57]$.

Aqueous leaf extracts of $A$. vera at doses of 200, 400, and $600 \mathrm{mg} / \mathrm{kg}$ and $A$. ferox at doses 500, 100, 200, and $400 \mathrm{mg} / \mathrm{kg}$ did not cause any toxic effects or mortality in all the treated animals [58-60]. Likewise, no toxic effects were observed when male Wistar rats were treated with an ethanolic extract of $A$. vera roots at doses of 100 , 200, and $400 \mathrm{mg} / \mathrm{kg}$ [61].

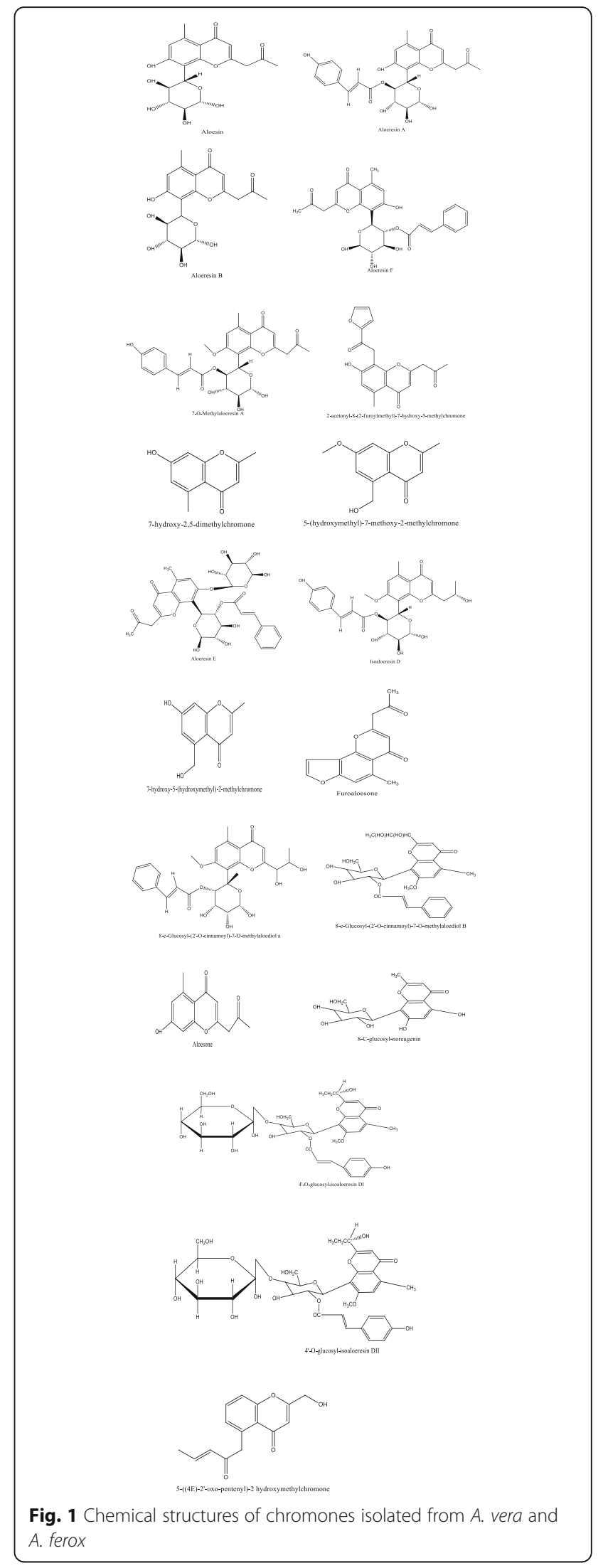




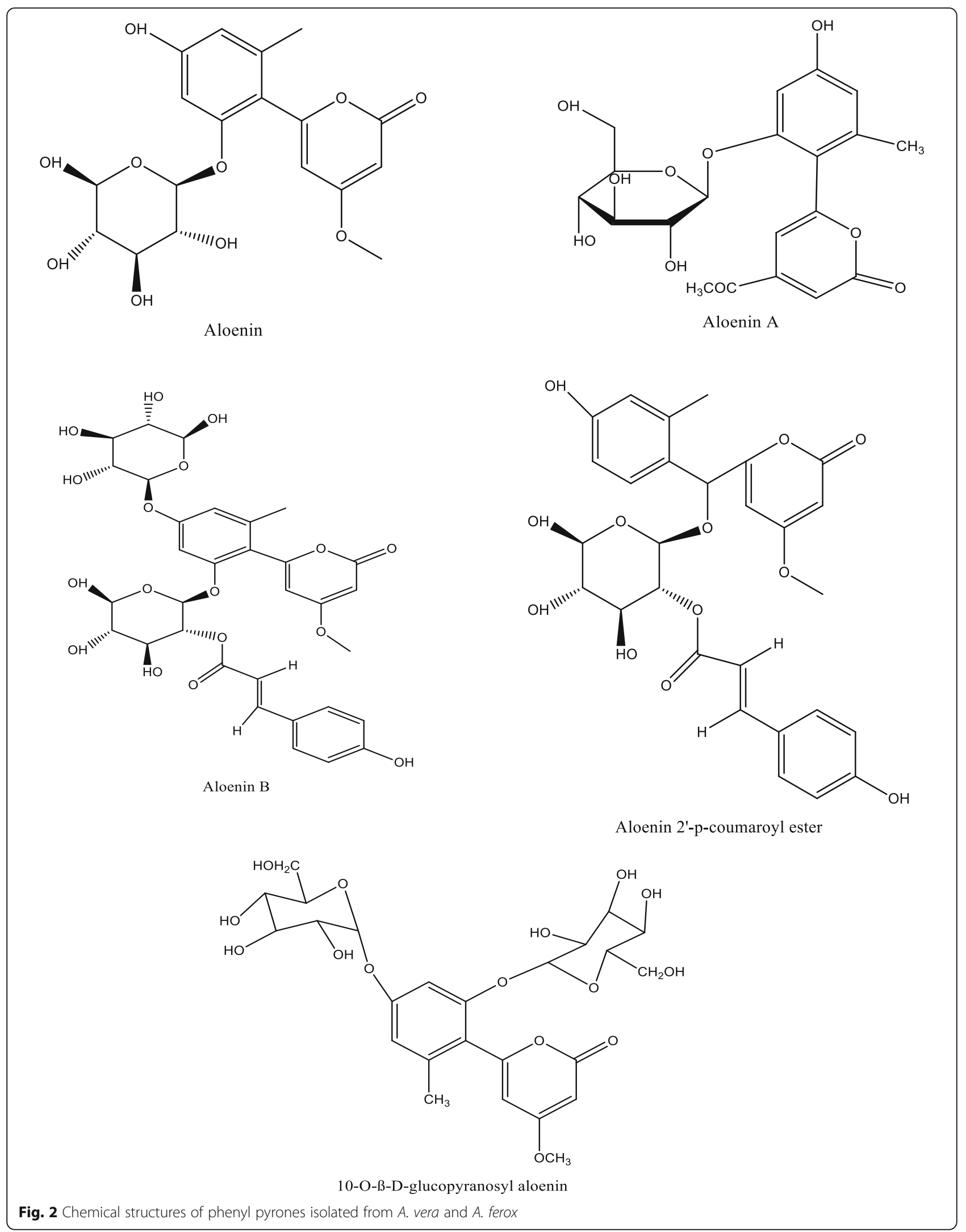



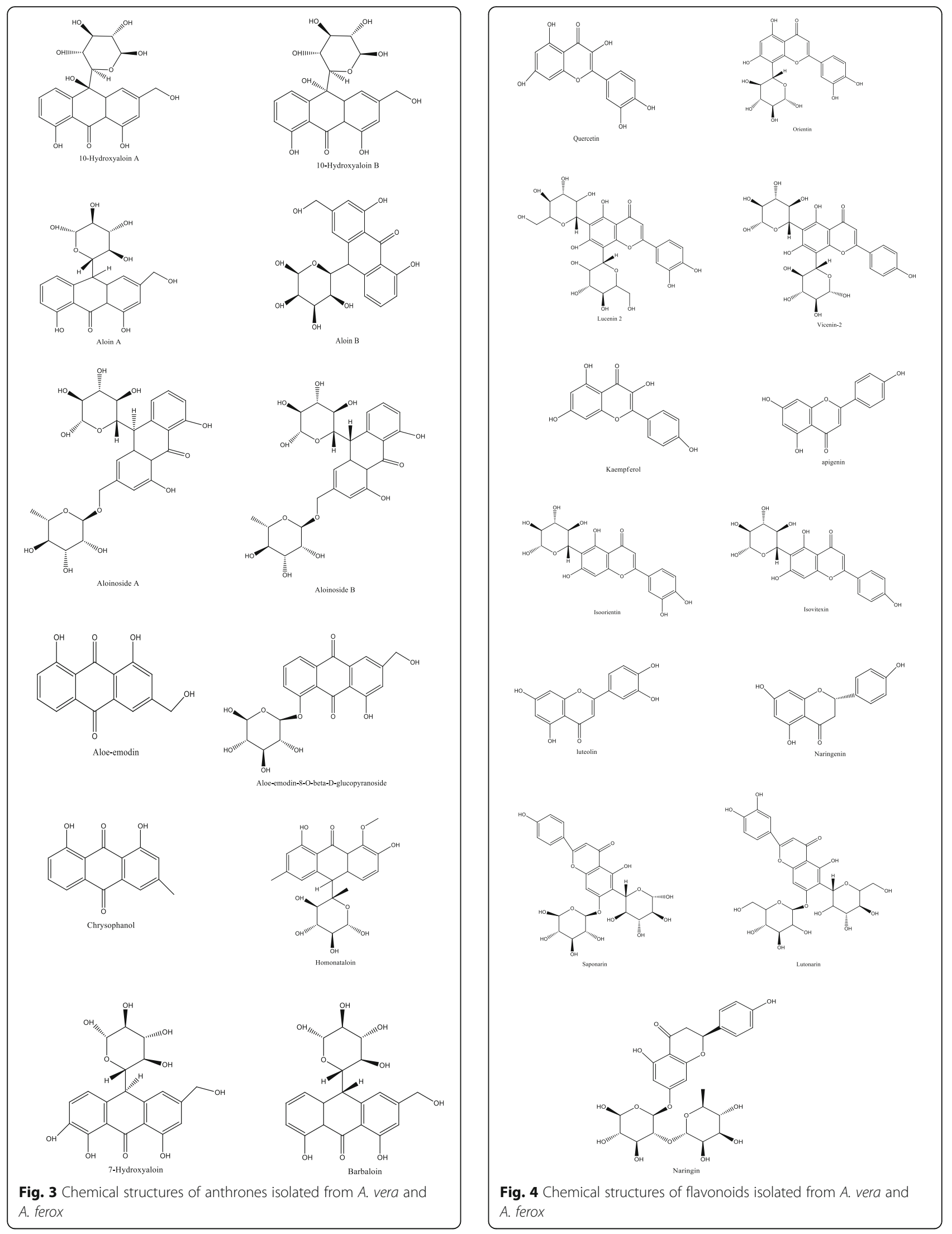


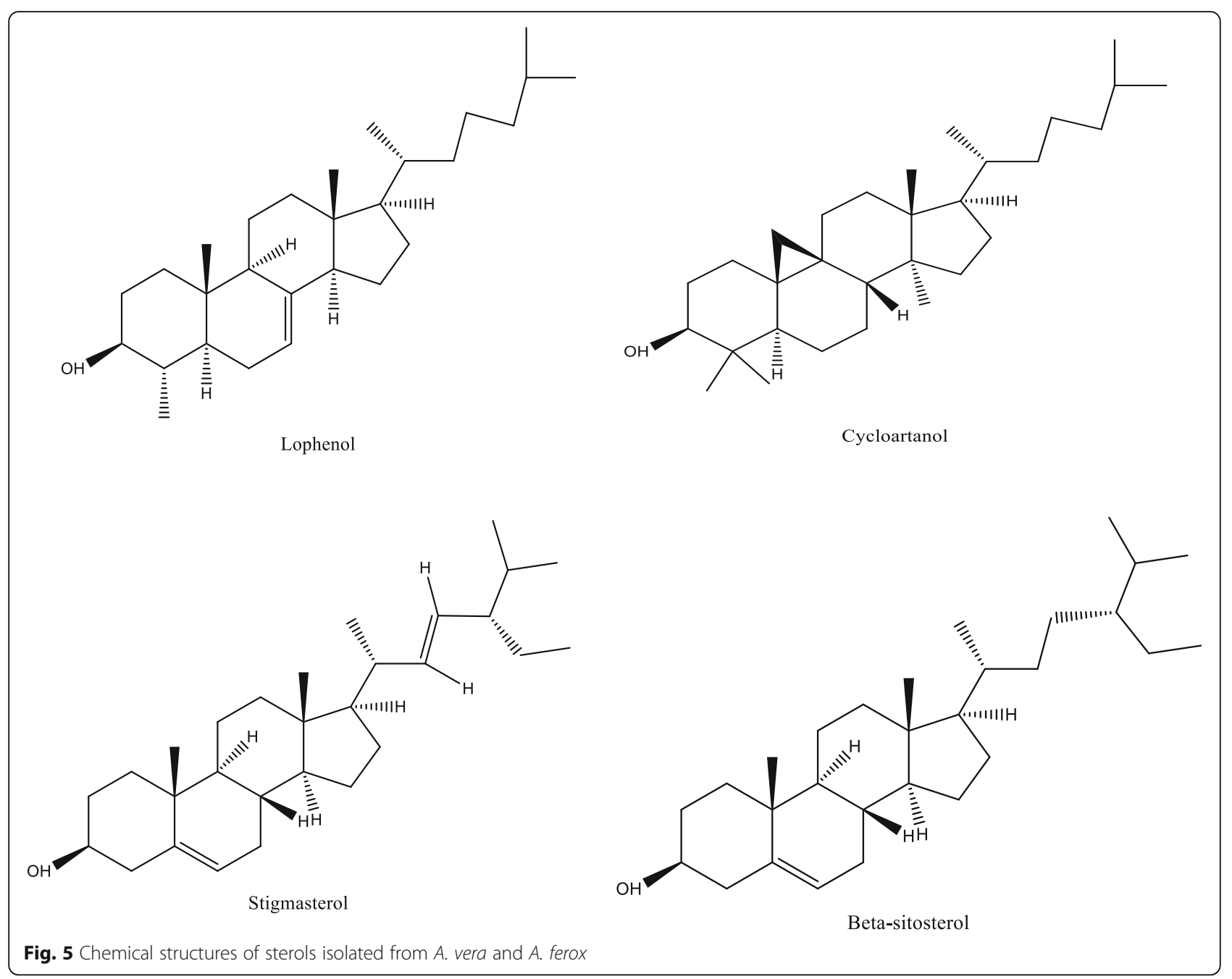

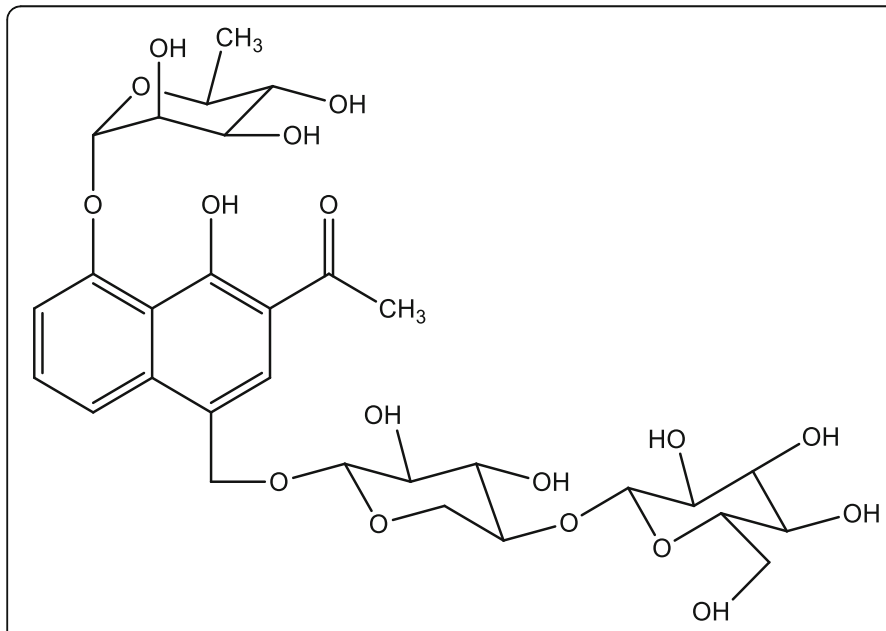

Aloveroside A<smiles>CC1CC(OC2OC(CO)C(O)C(O)C2O)Cc2cc(O)cc(O)c21</smiles>

Feroxin A

Fig. 6 Chemical structures of the naphthalene derivatives isolated from A. vera and A. ferox 


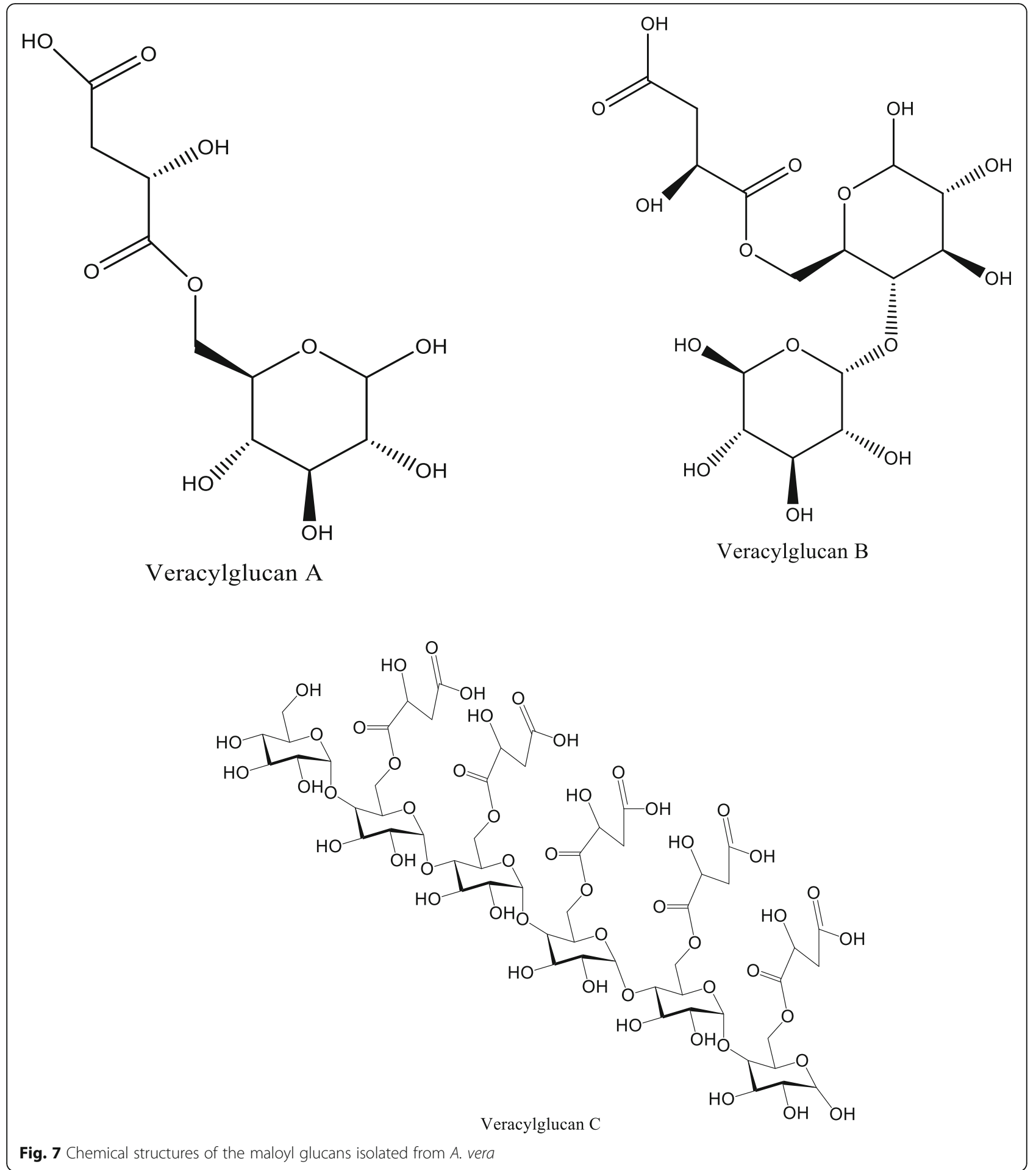

Ethanolic, acetone, and aqueous extracts of $A$. ferox roots and leaves caused death of nauplii of the brine shrimps at concentrations above $0.5 \mathrm{mg} / \mathrm{ml}$ [62]. Similarly, a herbal extract of $A$. vera at concentrations of $0.01,0.1$, and $1 \mathrm{mg} / \mathrm{ml}$ was toxic to the nauplii of the brine shrimps [63]. A hydroalcoholic extract of $A$. vera leaves caused mortality at 2560 $5120 \mathrm{mg} / \mathrm{kg}$ within $36-48 \mathrm{~h}$ in Kabir chicks [64]. A study by Shah et al. [65] revealed that an ethanolic extract of $A$. vera leaves caused reduced motor activity at doses of 1000 and $3000 \mathrm{mg} / \mathrm{kg}$ in male Swiss albino mice. 
<smiles>CCC(C)CC(O)CC</smiles>

5-methyl-3-heptanol

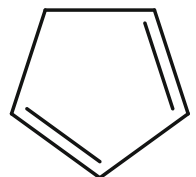

\section{1, 3-cyclopentadiene}

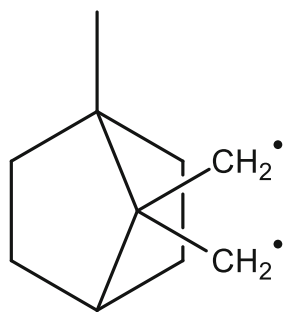

Bornylene

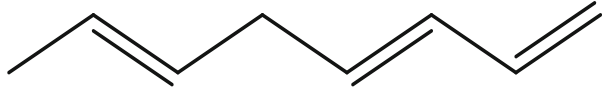

1,3, 6- octatriene

Fig. 8 Chemical structures of volatile oils isolated from A. ferox

\section{Subacute toxicity}

Administration of Aloe vera product (UP780), A. vera leaf juice, and gel for 14 days caused no harmful effects in rats and mice [58, 66, 67]. Wintola et al. [68] and Kwack et al. [69] reported similar results when $A$. vera leaf powder and $A$. ferox aqueous leaf extract were separately administered to rats.

A study by Koroye et al. [70] showed that administration of Aloe vera plus (GNLD) twice daily at volumes of $0.2,0.4$, and $0.8 \mathrm{~cm}^{3}$ for 14 and 28 days caused histological variations in the kidney tissues of the treated Wistar rats. A study by Sodani [71] displayed that the administration of $0.02 \mathrm{~cm}^{3}$ of $A$. vera leaf juice to male Swiss Webster mice over 21 days caused pathological effects on the kidneys.
In other studies, Aloe vera health drinks A and B administered over 28 days caused slight weight reduction and increase in white blood cell, red blood cell count, liver enzymes, serum urea, and creatinine levels in the rats given a volume of $1.0 \mathrm{~cm}^{3}$ [72]. A. vera leaf powder at a dose of 400,1200 , and $2000 \mathrm{mg} / \mathrm{kg}$ caused a significant reduction in white blood cell count and pigmentation of the kidneys in Sprague-Dawley rats [73].

Elevation in red blood cells, platelet count, hypertrophy of lungs, heart, and kidney and necrosis of spermatogenic cells was observed when an aqueous leaf extract of $A$. ferox at doses of 50,100, 200, and $400 \mathrm{mg} /$ $\mathrm{kg}$ was administered to Wistar rats for 14 days [59]. A decrease in the size of tubules, germ cell debris, and picnotic cells in the testes and testosterone was seen when

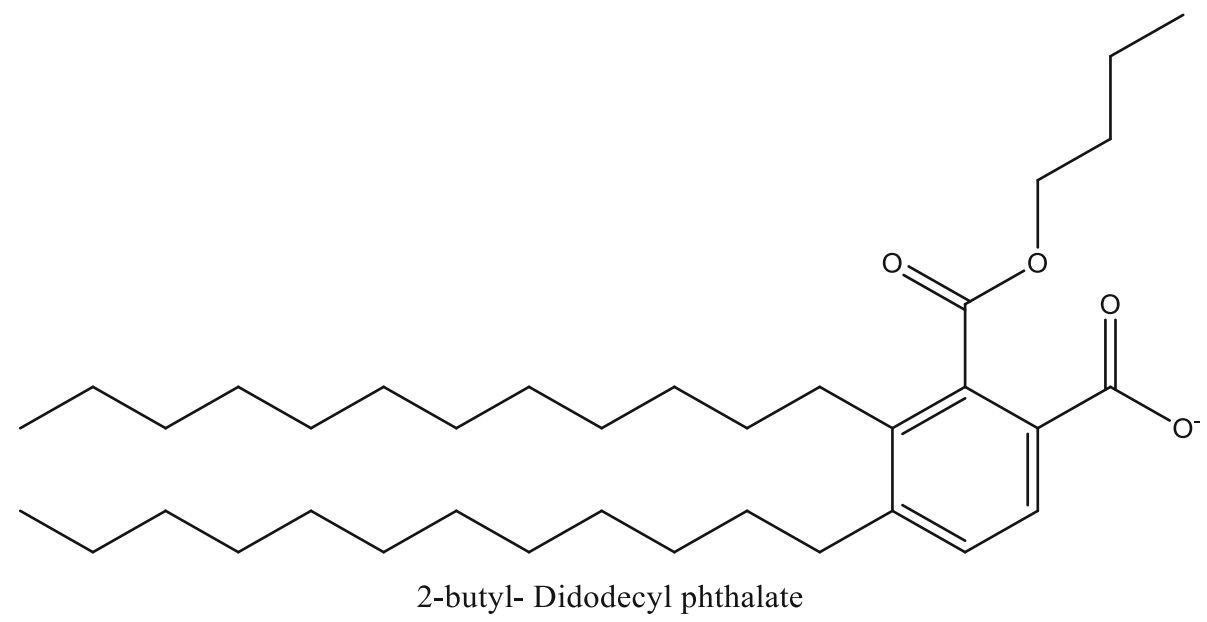

Fig. 9 Chemical structure of an ester isolated from $A$. vera 


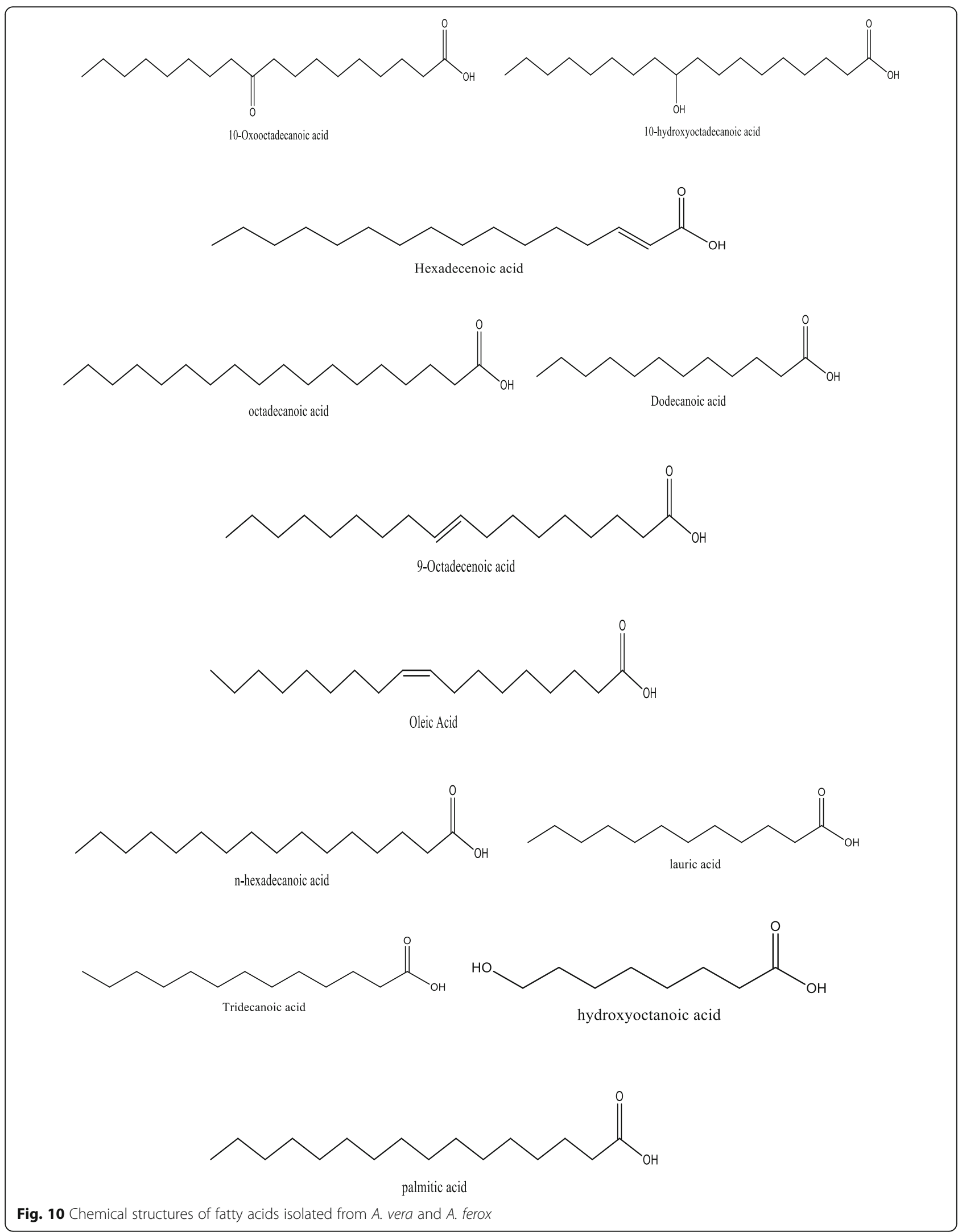


<smiles>O=C(/C=C/c1ccc(O)c(O)c1)OC1(C(=O)O)C[C@H](O)C(O)[C@H](O)C1</smiles><smiles>O=C(/C=C\c1ccc(O)cc1)O[C@@H]1C[C@@](O)(C(=O)O)C[C@H](O)[C@@H]1O</smiles><smiles>O=C(/C=C/c1ccc(O)c(O)c1)O[C@H]1C[C@@](O)(C(=O)O)C[C@H](O)[C@@H]1O</smiles><smiles>O=C(O)/C=C/c1ccc(O)c(O)c1</smiles><smiles>O=C(/C=C/c1ccc(O)c(O)c1)O[C@H]1C=C(C(=O)O)C[C@@H](O)[C@H]1O</smiles><smiles>O=C(O)c1cc(O)c(O)c(O)c1</smiles>
caffeoyl shikimic<smiles>COc1cc(/C=C/C(=O)O[C@@H]2C[C@](O)(C(=O)O)C[C@H](O)[C@H]2O)ccc1O</smiles><smiles>O=C(O)/C=C/c1ccc(O)cc1</smiles><smiles>COc1cc(/C=C/C(=O)O)ccc1O</smiles><smiles>O=C(O)/C=C/c1ccccc1</smiles>

Fig. 11 Chemical structures of phenolic acids isolated from A. vera and A. ferox 


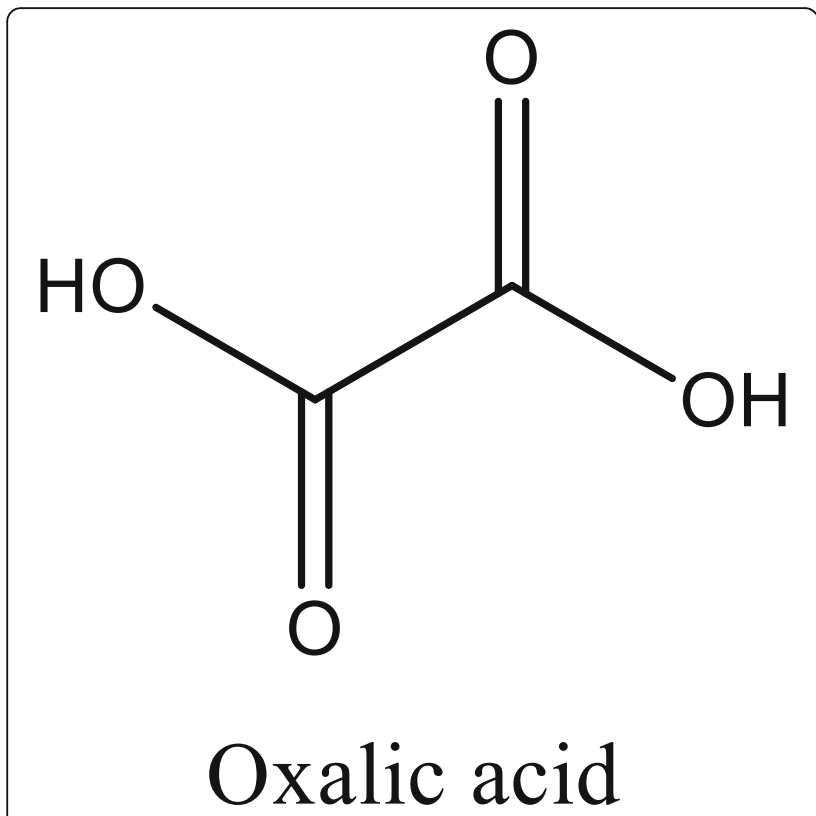

Fig. 12 Chemical structure of a dicarboxylic acid isolated from A. vera

A. vera gel product was administered for 28 days to male Swiss albino mice at the highest dose [74].

A study by Bala et al. [75] displayed that an aqueous gel extract of $A$. vera caused histopathological alterations in male Balb/c mice at 100 and $250 \mathrm{mg} / \mathrm{kg}$.

\section{Sub-chronic and chronic toxicity}

A study by Saritha and Anilakumar, [56] showed that administration of a methanolic gel extract of $A$. vera at doses of 1000, 2000, 4000, 8000, and $16000 \mathrm{mg} / \mathrm{kg}$ caused no mortalities or any changes in any of the investigated parameters at all the administered doses in the animals. Likewise, an aqueous leaf extract and supercritical carbon dioxide gel extract of $A$. vera caused no mortality or changes in the investigated parameters throughout the treatment period $[57,58,76]$.

A study by Mwale and Masika [59] showed that an aqueous leaf extract of A. ferox at doses of 50, 100, 200, and $400 \mathrm{mg} / \mathrm{kg}$ caused a rise in the red blood cells, monocytes, and platelets counts and also hypertrophy of lungs, heart, and kidney and necrosis of spermatogenic cells in rats at all doses.

An ethanolic gel extract of $A$. vera at a dose of 100 $\mathrm{mg} / \mathrm{kg}$ lowered the red blood cell count in addition to necrosis of the sex organs and hair loss around the genital area in male Swiss albino rats [65].

According to Koroye et al. [70], Aloe vera plus (GNLD) at doses of $0.2,0.4$, and $0.8 \mathrm{~cm}^{3}$ caused chronic inflammation, cell infiltration, necrosis, and fibrosis of the renal interstitium in all treated Wistar rats after 42 days of dosing.
Qmatrix ${ }^{\circ}$ a product from $A$. vera leaves also caused an increase in absolute and relative kidney weight of males at 500 and $2000 \mathrm{mg} / \mathrm{kg}$ [77].

A 2-year study showed that an aqueous nondecolorized leaf extract of $A$. vera was found to increase the rates of hyperplasia of the stomach, small intestines, large intestines, and mesenteric lymph nodes in both rats and mice [78].

\section{Toxic compounds in the Aloe vera and Aloe ferox}

Aloin, an anthraquinone present in both $A$. vera and $A$. ferox, has been associated with increased gastric motility causing diarrhea [79]. This explains why the Aloe species have been explored in relieving constipation. A study by Boudreau et al. [80] established that aloin caused pathological changes on the mucosa that were compared to those caused by Aloe vera whole leaf extract.

Aloe emodin, an anthraquinone present in $A$. vera, has been associated with hepatoxicity, genotoxicity, nephrotoxicity, phototoxicity, and reproductive toxicity [8185].

\section{Potential for treatment of COVID 19}

COVID 19 is caused by the Severe Acute Respiratory Syndrome coronavirus 2 (SARS-CoV-2). It belongs to RNA viruses and has four structural proteins (M (membrane), E (envelope), $\mathrm{N}$ (nucleocapsid), and $\mathrm{S}$ (spike)) [86]. The virus through its spike protein binds to the angiotensin-converting enzyme 2 (ACE2) receptors on the surface of the respiratory tract to facilitate its attachment and fusion with the host cell [86]. This is followed by entry into the host cell after priming of the $\mathrm{S}$ protein by the host cellular serine proteases TMPRSS2 [87]. The virus then releases its particles into the host cell, replicates, and invades the upper respiratory tract causing inflammation which later leads to acute respiratory distress. Treatment strategies involve use of antiviral drugs, immunomodulators, antibiotics, antioxidants, anti-inflammatory drugs, corticosteroids, and antipyretics [88-93]. Various medicinal plants including Aloe vera and Aloe ferox are being explored as potential drugs in the management of COVID 19 due to the various compounds they contain.

\section{Aloe vera}

In silico studies have shown that anthraquinones including chrysophanol, aloe emodin, aloeresin, aloin A \& B, 7-O-methylaloeresin, 9-dihydroxyl-2-O-(z)-cinnamoyl-7methoxy-aloesin, and isoaloeresin are potential SARSCoV-2 3CLpro protease inhibitors [94].

In addition, Aloe vera possesses anti-inflammatory activity $[42,60,95-100]$ which helps in preventing the release of pro-inflammatory markers that cause inflammation which induces acute respiratory distress, 
<smiles>C[C@@H]1O[C@H](Oc2c(-c3ccc(O)c(O)c3)oc3cc(O)cc(O)c3c2=O)[C@H](O)[C@@H](O)[C@@H]1O</smiles><smiles>Oc1cc(O)c2c(c1)OC(c1ccc(O)c(O)c1)C(O)C2</smiles><smiles>COc1cc(C(=O)O)ccc1O</smiles><smiles>COc1cc(/C=C/C(=O)O)cc(OC)c1O</smiles><smiles>Oc1ccc(/C=C/c2cc(O)cc(O)c2)cc1</smiles>

Resveratrol

Fig. 13 Chemical structures of phenolic compounds isolated from A. vera and A. ferox

the leading cause of mortality in COVID patients. Aloe vera also possesses immunomodulatory property [101104], which strengthens the immune system of the host hence curbing the spread of the infection.

In addition, $A$. vera contains a phytosterol, $\beta$ sitosterol, with immunostimulatory activity helping to reinforce the host's immune system. Molecular docking studies have shown that $\beta$-sitosterol strongly binds with the receptor-binding domain of the SARS-CoV-2 spike protein preventing the entry of the virus into the host cell [105].
Furthermore, Aloe vera contains mineral elements like zinc. Zinc has been found to inhibit the activity of corona RNA polymerase and SARS-coronavirus (SARSCoV-2) replication in cell culture studies [106].

\section{Aloe ferox}

In silico studies showed that anthraquinones (aloe emodin, aloinoside A, aloeresin D, Isoaloeresin A, etc.), phenolic compounds (pyrocatechol, p-Hydroxyacetophenone), and fatty acid derivatives (10-Hydroxyoctadecanoic acid, 10- 
<smiles>Cc1occ2c1C(=O)c1c(O)cccc1C2=O</smiles>

5-hydroxy-3-methylnaphtho[2,3-c] furan-4,9-dione<smiles>CC1=C2C(=O)c3c(O)cccc3C=C2CO1</smiles>

5-hydroxy-3-methylnaphtho[2,3-c] furan-4(1H)-one<smiles>Cc1occ2c1C(=O)c1c(O)cccc1C2</smiles>

5-hydroxy-3-methylnaphtho[2,3-c] furan-4(9H)-one

Fig. 14 Chemical structures of naphtho $[2,3-c]$ furan-4, 9-dione derivatives isolated from A. ferox 
<smiles>CC(C)=CCC/C(C)=C/CC/C(C)=C/CC/C=C(\C)CC/C=C(\C)CCC=C(C)C</smiles>

thymol<smiles>CC(=CCO)CCCC(C)CCCC(C)CCCC(C)C</smiles>

Phytol<smiles>C=C(C)[C@H]1CC[C@]2(C)CC[C@@]3(C)[C@@H](CC[C@]4(C)[C@]3(C)CC[C@]3(C)C(C)(C)C(O)CC[C@@]34C)[C@]12C</smiles>

Lupeol

Fig. 15 Chemical structures of some terpenoids isolated from A. vera and A. ferox

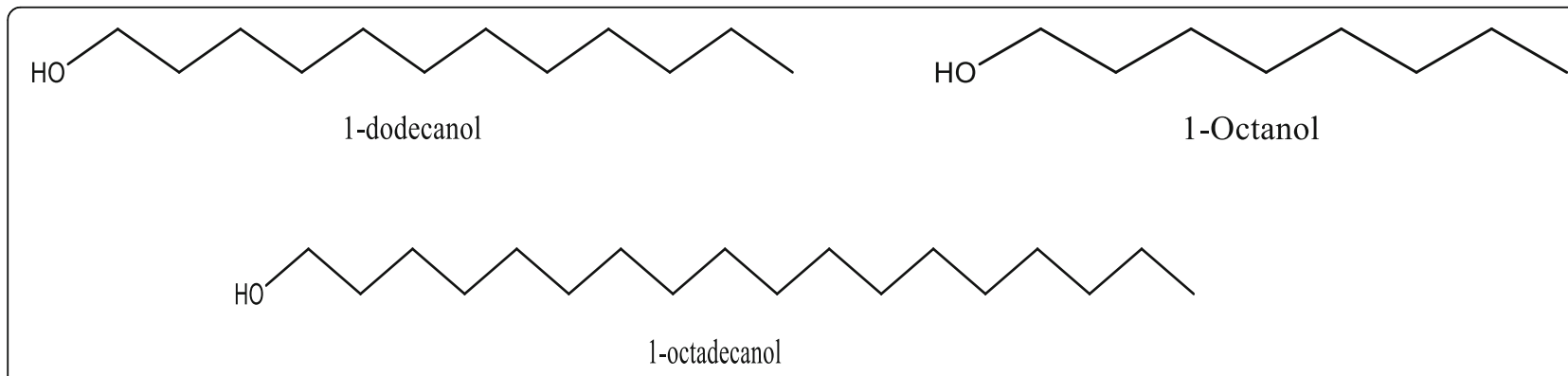

Fig. 16 Chemical structures of some alcohols isolated from A. vera and A. ferox 


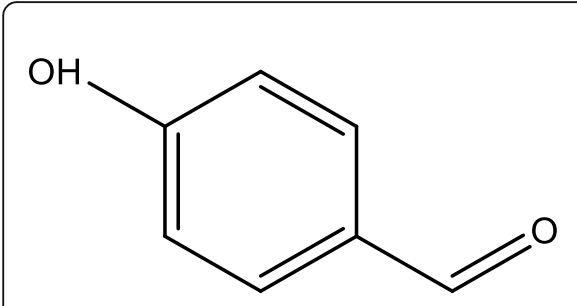

4-hydroxybenzaldehyde

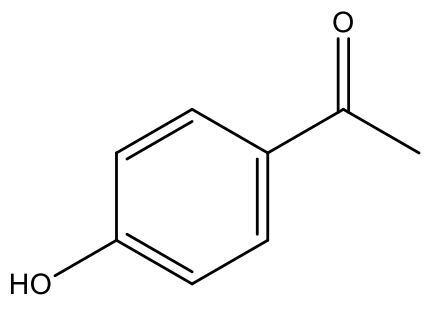

4'-Hydroxyacetophenone

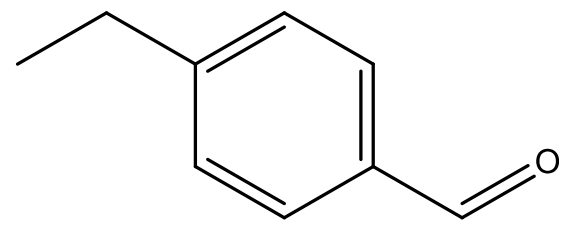

4-ethylbenzaldehyde

Fig. 17 Chemical structures of some aldehydes isolated from A. vera and A. ferox

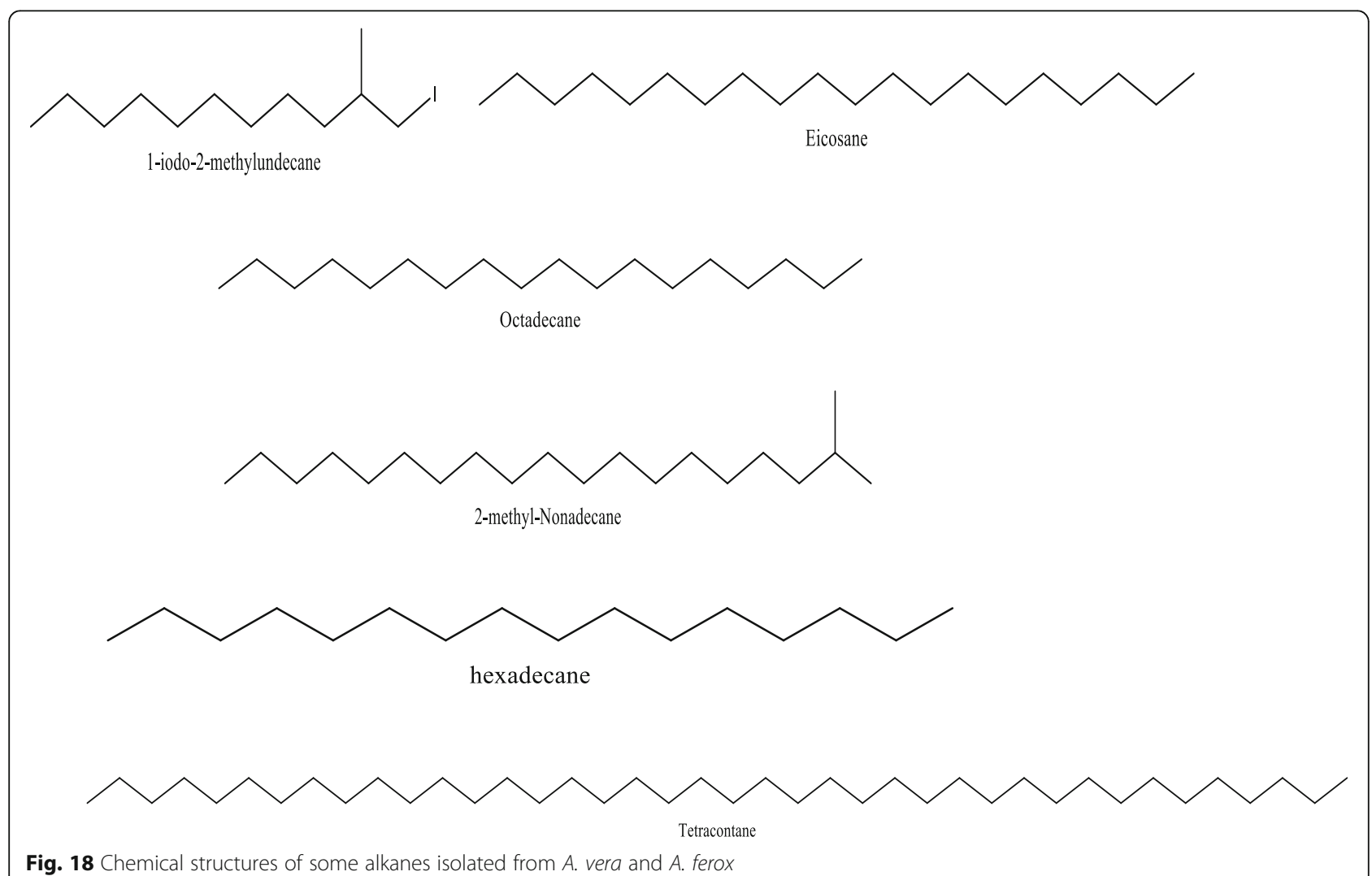

Fig. 18 Chemical structures of some alkanes isolated from A. vera and A. ferox 


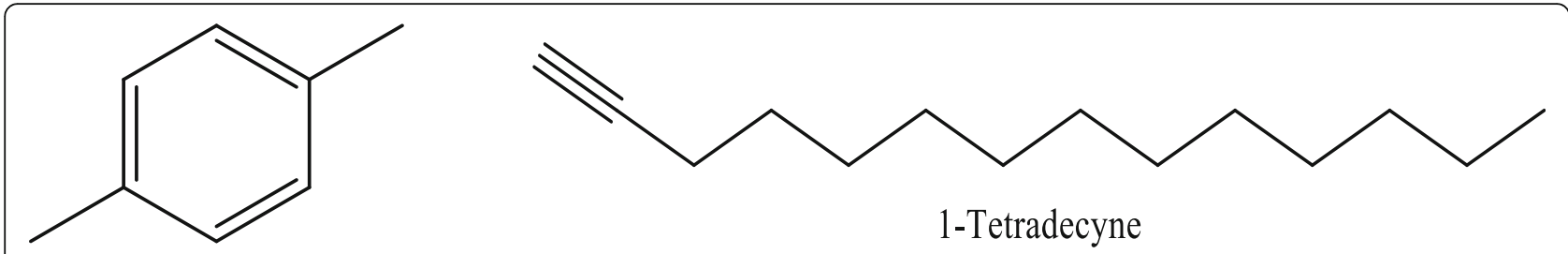

p-xylene

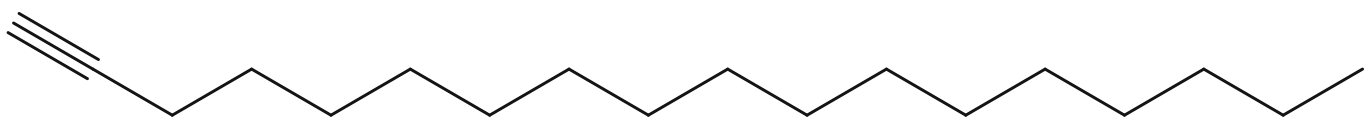

1-Octadecyne

Fig. 19 Chemical structures of some alkynes isolated from A. vera and A. ferox

Oxooctadecanoic acid) are potential SARS-CoV-2 main protease inhibitors [107].

Similar to $A$. vera, A. ferox is well endowed with antiinflammatory compounds $[108,109]$. These prevent the release of pro-inflammatory markers and cytokines that cause severe inflammation leading to acute respiratory distress in the patients.

\section{Conclusions}

$A$. vera and $A$. ferox contain vast phytochemicals including anthraquinones, flavonoids, and phytosterols, which can be further studied for activity against SARS-CoV-2. Since herbal preparations made from $A$. vera and $A$. ferox are currently sold, this information will be used by the regulatory authorities before they issue marketing approval to the manufacturers of these products. More toxicity studies need to be carried out on the aqueous extracts of $A$. vera and $A$. ferox since decoctions are the most commonly used preparations by the local population. Also, more studies need to be done on the isolated compounds from these species so that they can be excluded from the preparations in case they are found to be toxic.<smiles>Cc1c(C)c2c(c(C)c1O)CC[C@@](C)(CCC[C@H](C)CCC[C@H](C)CCCC(C)C)O2</smiles> 
Table 1 Phytochemical profile of whole leaves and flowers of Aloe vera

\begin{tabular}{|c|c|c|c|c|}
\hline $\begin{array}{l}\text { Plant } \\
\text { part }\end{array}$ & Phytochemicals present & $\begin{array}{l}\text { Method of extraction and } \\
\text { solvent used }\end{array}$ & $\begin{array}{l}\text { Method of } \\
\text { detection }\end{array}$ & Ref \\
\hline \multirow[t]{13}{*}{$\begin{array}{l}\text { Fresh } \\
\text { leaves }\end{array}$} & $\begin{array}{l}\text { Phenolic acids; caffeoylquinic acid hexoside and 3,4-O-(E) caffeoyl feruloyl quinic acid } \\
\text { Anthraquinones; Aloeresin E, isoaloeresin D, and 2'-O-feruloylaloesin } \\
\text { Flavonoids; Orientin, vicenin II, and Lucenin II }\end{array}$ & Cold percolation (methanol) & HPLC-MS & [12] \\
\hline & Phenols, Alkaloids, saponins, and sterols & Cold maceration (Hexane) & \multirow{4}{*}{$\begin{array}{l}\text { Phytochemical } \\
\text { screening and TLC }\end{array}$} & \multirow[t]{4}{*}{ [13] } \\
\hline & Saponins, sterols, and phenols & Cold maceration (Ethyl acetate) & & \\
\hline & Alkaloids, saponins, sterols, flavonoids, and phenols & Cold maceration (Methanol) & & \\
\hline & Alkaloids, tannins, sterols, flavonoids, and phenols & Cold maceration (Aqueous) & & \\
\hline & $\begin{array}{l}\text { Chromones; aloesin, 8-C-glucosyl-7-O-methyl-(S)-aloesol and isoaloeresin D. } \\
\text { Phenyl pyrones; aloenin and aloenin B } \\
\text { Anthrones; Aloe emodin, aloin A and B, 8-O-methyl-7-hydroxyaloin A and B, and 10-hydroxyaloin } \\
\text { A }\end{array}$ & Sonication (ethanol) & HPLC & [14] \\
\hline & $\begin{array}{l}\text { Sinapic acid, chlorogenic acid, aloin, aloe-emodin 8-O-beta-D-glucopyranoside, catechin, and } \\
\text { epicatechin }\end{array}$ & $\begin{array}{l}\text { Blended with } 80 \% \text { chilled } \\
\text { acetone }\end{array}$ & HPLC & [15] \\
\hline & $\begin{array}{l}\text { Cardiac glycosides, steroids, flavonoids, reducing sugar, phenolic compounds, terpenoids, } \\
\text { carbohydrates, amino acids, tannins, and saponin glycosides }\end{array}$ & $\begin{array}{l}\text { Cold maceration (methanol and } \\
\text { ethanol) } \\
\text { Hot maceration (water) }\end{array}$ & $\begin{array}{l}\text { Phytochemical } \\
\text { screening }\end{array}$ & [16] \\
\hline & $\begin{array}{l}\text { Dietary fiber (mannan), malic acid, a-tocopherol, phenolic compounds, and apigenin glycoside } \\
\text { derivatives }\end{array}$ & $\begin{array}{l}\text { Soxhlet extraction (petroleum } \\
\text { ether) } \\
\text { Maceration (ethanol: water) }\end{array}$ & Uv-vis, MS & [17] \\
\hline & Phytosterols ( $\beta$-sitosterol) & N/A & GC-MS & [18] \\
\hline & $\begin{array}{l}\text { Aldehydes; 4-ethyllbenzaldehyde and benzene acetaldehyde } \\
\text { Acids; lauric acid, palmitic acid } \\
\text { Carboxylic acids; hydroxyoctanoic acid derivative, octadecanoic acid } \\
\text { Alkanes; hexadecane derivative }\end{array}$ & Maceration (hexane) & GC-MS & [19] \\
\hline & $\begin{array}{l}\text { Terpenoids, Tannins, Flavonoids, resins, anthraquinones, saponins, glycosides, acidic compounds, } \\
\text { lignin, semi anthraquinone like derivatives, polysaccharides, vitamin B complex, phenol- } \\
\text { chromones, and chromones }\end{array}$ & Dissolution with $95 \%$ ethanol & $\begin{array}{l}\text { Phytochemical } \\
\text { screening and } \\
\text { HPLC }\end{array}$ & [20] \\
\hline & Alkaloids, anthraquinones, terpenes, phenols, tannins, coumarins, and flavonoids & $\begin{array}{l}\text { Sonication (dichloromethane } \\
\text { and methanol) }\end{array}$ & $\begin{array}{l}\text { Phytochemical } \\
\text { screening }\end{array}$ & [21] \\
\hline \multirow[t]{5}{*}{$\begin{array}{l}\text { Dried } \\
\text { leaves }\end{array}$} & $\begin{array}{l}\text { Alkaloids, phenols, flavonoids, saponins, glycosides, reducing sugars, phenolic compounds, } \\
\text { tannins, steroids, and terpenoids }\end{array}$ & Cold percolation (methanol) & $\begin{array}{l}\text { Phytochemical } \\
\text { screening }\end{array}$ & [22] \\
\hline & $\begin{array}{l}\text { Flavonoids, tannins, and saponins } \\
\text { Terpenoids; Squalene, phytol, and lupeol } \\
\text { Alkynes; } 1 \text {-Tetradecyne and 1-Octadecyne } \\
\text { Carboxylic acids; Tridecanoic acid and } \\
\text { n-Hexadecanoic acid } \\
\text { Alkanes; } 1 \text {-lodo-2-methylundecane, eicosane, octadecane, 2-methyl nonadecane, and tetracon- } \\
\text { tane, 3,5,24-trimethyl-C } \\
\text { Fatty acids; Oleic acid } \\
\text { Dicarboxylic acid; Oxalic acid } \\
\text { Alcohol; } 1 \text {-Octanol } \\
\text { Ester; 2-butyl- didodecyl phthalate } \\
\text { Vitamins; a-Tocopherol and vitamin E } \\
\text { Sterols; } \beta \text {-Sitosterol }\end{array}$ & $\begin{array}{l}\text { Soxhlet extraction (distilled } \\
\text { water, ethanol, acetone } \\
\text { solution) }\end{array}$ & $\begin{array}{l}\text { Phytochemical } \\
\text { screening and GC- } \\
\text { MS }\end{array}$ & [23] \\
\hline & $\begin{array}{l}\text { Anthraquinones, tannins, flavonoids, saponins, squalene, oleic acid, dodecanoic acid, p-xylene, and } \\
\text { n-hexadecanoic acid }\end{array}$ & Maceration (Water) & $\begin{array}{l}\text { Phytochemical } \\
\text { screening and GC- } \\
\text { MS }\end{array}$ & [24] \\
\hline & $\begin{array}{l}\text { Saponins, phytosterols, terpenoids, alkaloids, flavonoids, carbohydrates, proteins, phenols, and } \\
\text { carbohydrates }\end{array}$ & $\begin{array}{l}\text { Soxhlet extraction }(80 \% \\
\text { ethanol) }\end{array}$ & $\begin{array}{l}\text { Phytochemical } \\
\text { screening and GC- } \\
\text { MS }\end{array}$ & [25] \\
\hline & tannins, flavonoids, terpenoids, carbohydrates, and alkaloids & $\begin{array}{l}\text { Soxhlet extraction (chloroform) } \\
\text { Maceration (water) }\end{array}$ & $\begin{array}{l}\text { Phytochemical } \\
\text { screening }\end{array}$ & [26] \\
\hline \multirow[t]{3}{*}{$\begin{array}{l}\text { Dried } \\
\text { Flowers }\end{array}$} & Phenolic compounds; Quercitrin, gentisic acid, and epicatechin & Maceration (methanol) & $\begin{array}{l}\text { Reverse Phase- } \\
\text { HPLC }\end{array}$ & [27] \\
\hline & $\begin{array}{l}\text { Coumarin, gallic acid, caffeic acid, D-catechin, vanillic acid, narigenin, resveratrol, cinnamic acid, } \\
\text { thymol, quercetin, and naringin }\end{array}$ & Maceration (70\% ethanol) & HPLC & [28] \\
\hline & $\begin{array}{l}\text { Phenolic acids; Chlorogenic, caffeic, 5-p-coumaroylquinic, caffeoyl shikimic, 5-feruloyl quinic, 5-p- } \\
\text { cis-coumaroylquinic, p-coumaric, and ferulic acids } \\
\text { Flavonoids; luteolin, apigenin, quercetin, isoorientin, isovitexin, kaempferol, saponarin, and } \\
\text { lutonarin } \\
\text { Anthranoids; Aloe emodin }\end{array}$ & Ultrasonication (methanol) & $\begin{array}{l}\text { HPLC-DAD and } \\
\text { HPLC-MS/MS }\end{array}$ & [29] \\
\hline
\end{tabular}


Table 2 Phytochemical profile of the gel, skin, powder, and extracts from A. vera leaves

\begin{tabular}{|c|c|c|c|c|}
\hline $\begin{array}{l}\text { Plant } \\
\text { preparation } \\
\text { used }\end{array}$ & Phytochemicals present & $\begin{array}{l}\text { Method of } \\
\text { extraction and } \\
\text { solvent used }\end{array}$ & Method of detection & Ref \\
\hline $\begin{array}{l}\text { Crude herbal } \\
\text { extract }\end{array}$ & $\begin{array}{l}\text { Alkaloids, free anthraquinones, amino acids, saponins, tannins, } \\
\text { triterpenoids, steroids, glycosides, and flavonoids }\end{array}$ & N/A & $\begin{array}{l}\text { Phytochemical screening and } \\
\text { TLC }\end{array}$ & {$[30]$} \\
\hline $\begin{array}{l}\text { Ethanol } \\
\text { herbal } \\
\text { extract }\end{array}$ & $\begin{array}{l}\text { 6-phenyl-2-pyrone derivatives ( } p \text {-coumaroyl aloenin and aloenin A), } \\
\text { naphthalene derivatives (aloveroside A), and anthraquinones. }\end{array}$ & N/A & TLC, HPLC, MS, IR, and NMR & [31] \\
\hline \multirow[t]{2}{*}{ Leaf exudate } & $\begin{array}{l}\text { Homonataloin, aloesin, aloenin, barbaloin, aloinosides A\&B, and } \\
\text { aloesone }\end{array}$ & $\begin{array}{l}\text { Exudation into } \\
\text { methanol }\end{array}$ & TLC & [32] \\
\hline & $\begin{array}{l}\text { Chromones; aloesin, 8-C-glucosyl-(R)-aloesol, 8-C-glucosyl-7-O-(S)- } \\
\text { methylaloesol, and }\end{array}$ & $\begin{array}{l}\text { Ultrasonic extraction } \\
\text { (methanol and water) }\end{array}$ & HPLC-DAD and LCMS-IT-TOF & [33] \\
\hline
\end{tabular}

5-((S)-2ß-oxo-4'-hydroxypentyl-2(-glucopyranosyl-oxymethyl)

chromone.

Phenyl pyrones; 10-O-d-glucopyranosyl aloenin, aloenin, aloenin B,

and aloenin-2'-p-coumaroyl ester

Anthrones; 10-hydroxyaloin B, 10-hydroxyaloin A, aloin B, aloin A,

aloinoside $B$, and aloinoside $A$

Anthraquinone; Aloe emodin

Naphthalene derivative; Aloveroside B

Free and glycosylated chromones: Aloesin and aloeresin A

Anthraquinones: Aloin and aloe emodin

Sonication

Colorimetric assays, TOF-MS

Saponins, flavonoids, and tannins

ethyl acetate)

Soxhlet (petroleum

ether: chloroform:

ethanol)

Leaf gel

Fatty acids; hexadecanoic acid, octadecanoic acid, and 9-octadenoic acid

Maceration (ethanol) GC-MS

Phytochemical screening

Sterols: Sitosterol, and stigmasterol

Alcohols; 1-octadecanol, 1-dodecanol

Alkanes; debocane, tricosane, and 4-methyl, 1-

(phenylthioxomethyl)piperidine

Chromones; 8-C-glucosyl-(2'-O-cinnamoyl)-7-O-methylaloediol A and

B, 8-C-glucosyl-noreugenin, 4'-O-glucosyl-isoaloeresin DII, and 4'-O-

glucosyl-isoaloeresin DI

(Ethanol)

HPLC and NMR

Phytosterols; cycloartanol, lophenol, 24-ethyl-lophenol, 24-methyllophenol, and 24-methylene-cycloartanol

Maloyl glucans; Veracylglucan A, B, and C

Pyrocatechol, ascorbic acid, coumaric acid, and p-coumaric acid

Alkaloids, aldehydes, phytosterols, pyrimidines, phenolic acids/ polyphenols, fatty acids, alkanes, organic acids, alcohols, dicarboxylic acids, ketones, and indoles

Trichloromethane and methanol

Cold maceration (ethanol and methanol)

Blended with 95\%

ethanol and

centrifuged

Carbohydrates, resins, reducing sugars, glucuronic acid, pentose derivatives, acetylated mannan, galactoglucoarabinomannan, glucomannone, and monosaccharides (alverose)

Cardiotonic glycosides, anthraglycosides, mucilages, and reducing sugars

Sterols type $\Delta^{5}$ and anthraquinones

Triterpenoids, carbohydrates, saponins, anthraquinones, and naphthoquinones

Leaf skin

Steroids, tannins, terpenoids, catechin, carotenoids, and anthraquinones

Phenolic compounds; Sinapic acid, catechin, and quercetin

Leaf Powder

Chromones; Aloenin B, 5-(hydroxymethyl)-7-methoxy-2-
Extraction with ethanol

Extraction with water Soxhlet (Chloroform)

Soxhlet extraction (Ethanol)

Maceration (ethanol)

Maceration
(methanol)

Ultrasonication $(70 \%$ methanol) methylchromone, aloin A \& B, aloe emodin, 5-((4E)-2'-oxo-pentenyl)-2

Column chromatography, NMR

NMR, ESIMS, MALDI-TOF-MS, and capillary electrophoresis.

Solvent fractionation, TLC, and GC-MS

GC-MS

Phytochemical screening and HPLC

Phytochemical screening

Phytochemical screening

Phytochemical screening

Phytochemical screening

RP-HPLC

UV, IR, 1D and 2D NMR, and High-Resolution Mass 
Table 2 Phytochemical profile of the gel, skin, powder, and extracts from A. vera leaves (Continued)

\begin{tabular}{llll}
\hline $\begin{array}{l}\text { Plant } \\
\text { preparation } \\
\text { used }\end{array}$ & Phytochemicals present & $\begin{array}{l}\text { Method of } \\
\text { extraction and } \\
\text { solvent used }\end{array}$ & Method of detection \\
\hline & $\begin{array}{l}\text { hydroxymethylchromone, 7-hydroxy-5-(hydroxymethyl)-2-methylchro- } \\
\text { mone, and 10-hydroxyaloin A \&B }\end{array}$ & Spectrometry (HRMS) \\
& $\begin{array}{l}\text { Aloeveraside A and B, benzene derivatives, terpenoids, } \\
\text { Resin } \\
\text { anthraquinones, coumarins, anthraquinone glycosides, quinones, }\end{array}$ & $\begin{array}{l}\text { Cold maceration } \\
\text { (methanol) }\end{array}$ & TLC, NMR, IR, and MS \\
& & & \\
\hline
\end{tabular}

Table 3 Phytochemical profile of Aloe ferox

\begin{tabular}{|c|c|c|c|c|}
\hline $\begin{array}{l}\text { Plant part/ } \\
\text { preparation } \\
\text { used }\end{array}$ & Phytochemicals present & Method of extraction and solvent used & Method of detection & Ref \\
\hline \multirow[t]{2}{*}{ Fresh leaves } & $\begin{array}{l}\text { Phenolic acids; caffeoylquinic acid hexoside and 3,4- } \\
\text { O-(E) caffeoyl feruloyl quinic acid } \\
\text { Anthraquinones; Aloeresin E, isoaloeresin D, and 2'-O- } \\
\text { feruloylaloesin } \\
\text { Flavonoids; Lucenin II, vicenin II, and orientin }\end{array}$ & Cold percolation (methanol) & HPLC-MS & {$[12]$} \\
\hline & $\begin{array}{l}\text { Sinapic acid, catechin, chlorogenic acid, aloe-emodin- } \\
\text { 8-O-beta-D-glucopyranoside, aloin, and epicatechin }\end{array}$ & Blended with $80 \%$ chilled acetone & HPLC & [15] \\
\hline \multirow[t]{3}{*}{ Dried leaves } & Aloe emodin, aloin A, and chrysophanol & Maceration (water) & $\begin{array}{l}\text { Vacuum liquid } \\
\text { fractionation, column } \\
\text { chromatography }\end{array}$ & [47] \\
\hline & $\begin{array}{l}\text { Phenols, saponins, alkaloids, flavonoids, } \\
\text { proanthocyanidins, flavonols, and tannins }\end{array}$ & $\begin{array}{l}\text { Cold maceration (distilled water, acetone, } \\
\text { methanol, and ethanol) }\end{array}$ & $\begin{array}{l}\text { Phytochemical } \\
\text { screening }\end{array}$ & [48] \\
\hline & Condensed tannins, flavonoids, and gallotannins & $\begin{array}{l}\text { Extraction by sonication (methanol) followed } \\
\text { by successive extraction with petroleum } \\
\text { ether, dichloromethane, and ethanol) }\end{array}$ & $\begin{array}{l}\text { Phytochemical } \\
\text { screening }\end{array}$ & [49] \\
\hline $\begin{array}{l}\text { Dried leaf } \\
\text { latex }\end{array}$ & $\begin{array}{l}\text { Naphtha }[2,3-c] \text { furan derivatives; 5-hydroxy-3-methyl- } \\
\text { naphtho[2,3-c] furan-4,9-dione and 5-hydroxy-3-methyl- } \\
\text { naphtho[2,3-c] furan-4(1H)-one, anthraquinones, and 5- } \\
\text { hydroxy-3-methylnaphtho[2,3-c] furan-4(9H)-one }\end{array}$ & Dissolution in water & $\begin{array}{l}\text { X-ray analysis and } \\
\text { spectroscopy }\end{array}$ & [50] \\
\hline Leaf resin & hydroxyanthracene derivatives (aloin) & N/A & TLC & [51] \\
\hline Leaf juice & $\begin{array}{l}\text { Volatile oils; 5-methyl-3-heptanol, bornylene, 1, 3- } \\
\text { cyclopentadiene, 3, } 6 \text { octatriene, and 3-cyclohexane-1- } \\
\text { hetanol }\end{array}$ & Hydro distillation (water) & GC-MS & [52] \\
\hline \multirow[t]{2}{*}{$\begin{array}{l}\text { Dried } \\
\text { exudate }\end{array}$} & $\begin{array}{l}\text { Free and glycosylated chromones; Aloeresin B \& F } \\
\text { and 7-O-methyl aloeresin } \\
\text { Naphthalene derivative; feroxin A } \\
\text { Anthraquinones; hydroxyaloin and 8-O-Methyl- 7- } \\
\text { hydroxyaloin }\end{array}$ & $\begin{array}{l}\text { Sonication (methanol, acetone, and ethyl } \\
\text { acetate mixture) }\end{array}$ & $\begin{array}{l}\text { Colorimetric assays, Q- } \\
\text { TOF-MS }\end{array}$ & [34] \\
\hline & $\begin{array}{l}\text { Aloe emodin, furoaloesone, } \text {-hydroxybenzaldehyde, } \\
\text { 10-oxooctadecanoic acid, } \text {-hydroxyacetophenone, py- } \\
\text { rocatechol, 7-hydroxy-2,5-dimethylchromone, 10- } \\
\text { hydroxyoctadecanoic acid, 2-acetonyl-8-(2-furoyl- } \\
\text { methyl)-7-hydroxy-5-methylchromone, and methyl 10- } \\
\text { hydroxyoctadecanoate, }\end{array}$ & Maceration (hexane and aqueous acetone) & $\begin{array}{l}\text { Solvent partitioning, } \\
\text { column } \\
\text { chromatography, TLC, } \\
\text { NMR, and MS }\end{array}$ & [53] \\
\hline Roots & $\begin{array}{l}\text { Phenols, alkaloids, flavonoids, tannins, flavonols, and } \\
\text { saponins }\end{array}$ & Maceration (water) & $\begin{array}{l}\text { Phytochemical } \\
\text { screening }\end{array}$ & [54] \\
\hline Leaf gel & $\begin{array}{l}\text { Alkaloids, phenolic acids/polyphenols, phytosterols, } \\
\text { organic acids, fatty acids, indoles, alkanes, alcohols, } \\
\text { pyrimidines, aldehydes, dicarboxylic acids, and ketones }\end{array}$ & Blended with $95 \%$ ethanol and centrifuged & GC-MS & {$[55]$} \\
\hline
\end{tabular}




\section{Abbreviations}

ESIMS: Electrospray ionization mass spectrometry; GC-MS: Gas chromatography-mass spectrometry; HPLC: High-performance liquid chromatography; HPLC-DAD: High-performance liquid chromatography with a diode-array detector; HPLC-MS: High-performance liquid chromatographymass spectrometry; MALDI-TOF-MS: Matrix-assisted laser desorption/ ionization-time of flight; MS : Mass spectrometry; NMRS: Nuclear magnetic resonance spectrometry; TLC: Thin-layer chromatography; TOF-MS: Triple quadrupole and time-of-flight mass spectrometry

\section{Acknowledgements}

We would like to thank Mr Emanuel L Peter for the help rendered in the preparation of the manuscript.

\section{Authors' contributions}

FN conceived the research idea, collected the data and prepared the first draft of the manuscript. JO and POE screened for duplication and also carried out data analysis. IK drew all the structures in the manuscript. All the authors read and approved the final manuscript

\section{Funding}

Not applicable.

\section{Availability of data and materials}

Not applicable.

\section{Declarations}

Ethics approval and consent to participate

Not applicable.

\section{Consent for publication}

Not applicable.

\section{Competing interests}

The authors declare that they have no competing interests.

\section{Author details}

${ }^{1}$ Pharm-Bio Technology and Traditional Medicine Centre of Excellence, Mbarara University of Science and Technology, Mbarara, Uganda. 2Department of Pharmaceutical Sciences, Faculty of Medicine, Mbarara University of Science and Technology, P.O. Box 1410, Mbarara, Uganda. ${ }^{3}$ Department of Pharmacology and Therapeutics, Faculty of Medicine, Mbarara University of Science and Technology, Mbarara, Uganda. ${ }^{4}$ Department of Pharmacy, Faculty of Medicine, Mbarara University of Science and Technology, Mbarara, Uganda.

Received: 16 March 2021 Accepted: 5 July 2021

Published online: 21 July 2021

\section{References}

1. Chikezie PC, Ojiako OA (2015) 'Herbal medicine: yesterday, today and tomorrow' Altern Integr Med 4(3):195. https://doi.org/10.4172/2327-5162.1 000195

2. Salehi B, Albayrak S, Antolak H, Kręgiel D, Pawlikowska E, Sharifi-Rad M, Uprety Y, Tsouh Fokou PV, Yousef Z, Amiruddin Zakaria Z, Varoni EM (2018) Aloe genus plants: from farm to food applications and phytopharmacotherapy. Int J Mol Sci 19(9):2843 https://doi.org/10.3390/ ijms19092843

3. Surjushe A, Vasani R, Saple DG (2008) Aloe vera: a short review. Indian J Dermatol 53(4):163-166. https://doi.org/10.4103/0019-5154.44785

4. Sharma P, Kharkwal AC, Kharkwal H, Abdin MZ, Varma A (2014) A review on pharmacological properties of Aloe vera. Int J Pharm Sci Rev Res 29(2):31-37

5. Banik S, Sharangi AB (2019) Phytochemistry, health benefits and toxicological profile of Aloe. J Pharmacog Phytochem 8(3):4499-4506

6. Cock IE (2015) The genus Aloe: phytochemistry and therapeutic use including treatments for gastrointestinal conditions and chronic inflammation. Prog Drug Res 70:179-235. https://doi.org/10.1007/978-3-034 8-0927-6_6
7. Guo X, Mei N (2016) Aloe vera: a review of toxicity and adverse clinical effects. J Environ Sci Health C 34(2):77-96. https://doi.org/10.1080/1 0590501.2016 .1166826

8. Haq I (2004) Safety of medicinal plants. Pak J Med Res 43(4):203-210

9. Ernst E (2003) Cardiovascular adverse effects of herbal medicines: a systematic review of the recent literature. Can J Cardiol 19(7):818-827

10. Emmanuel AM, Roger KK, Toussaint DG, Koffi K (2018) Acute and subacute toxicity of the aqueous extract of Amaranthus viridis (Amaranthaceae) leaves in rats. J Phytopharmacolo 7(4):366-372

11. de Mel Y, Perera S, Ratnaweera PB, Jayasinghe CD (2017) Novel insights of toxicological evaluation of herbal medicine: human based toxicological assays. Asian J Pharm Pharmacol 3(2):41-49

12. El Sayed AM, Ezzat SM, El Naggar MM, El Hawary SS (2016) In vivo diabetic wound healing effect and HPLC-DAD-ESI-MS/MS profiling of the methanol extracts of eight Aloe species. Rev Bras 26(3):352-362. https://doi.org/10.101 6/j.bjp.2016.01.009

13. Dharajiya D, Pagi N, Jasani H, Patel P (2017) Antimicrobial activity and phytochemical screening of Aloe vera (Aloe barbadensis Miller). Int J Curr Microbiol App Sci 6(3):2152-2162

14. Park MK, Park JH, Kim NY, Shin YG, Choi YS, Lee JG, Kim KH, Lee SK (1998) Analysis of 13 phenolic compounds in Aloe species by high performance liquid chromatography. Int J Plant Chem Biochem Techn 9(4):186-191

15. Lai Q, Wang H, Guo X, Abbasi AM, Wang T, Li T, Fu X, Li J, Liu RH (2016) Comparison of phytochemical profiles, antioxidant and cellular antioxidant activities of seven cultivars of Aloe. Int J Food Sci Technol 51(6):1489-1494. https://doi.org/10.1111/ijfs.13093

16. Malik NZ, Riaz M, Noshad QQ, Rashid N, Ain QU, Hussain A (2017) Morphological, phytochemical and antifungal analysis of Aloe vera L. leaf extracts. Asian J Agri Biol 5(4):177-187

17. Añibarro-Ortega M, Pinela J, Barros L, Cirić A, Silva SP, Coelho E, Mocan A, Calhelha RC, Soković M, Coimbra MA, Ferreira IC (2019) Compositional features and bioactive properties of Aloe vera leaf (fillet, mucilage, and rind) and flower. Antioxidants 8(10):1-21

18. Palermo FA, Cocci P, Angeletti M, Felici A, Polzonetti-Magni AM, Mosconi G (2013) Dietary Aloe vera components' effects on cholesterol lowering and estrogenic responses in juvenile goldfish, Carassius auratus. Fish Physiol Biochem 39(4):851-861. https://doi.org/10.1007/s10695-012-9745-7

19. Dey P, Dutta S, Chowdhury A, Das AP, Chaudhuri TK (2017) Variation in phytochemical composition reveals distinct divergence of Aloe vera $(\mathrm{L}$.) Burm. F. From other aloe species: rationale behind selective preference of Aloe vera in nutritional and therapeutic use. J Evid Based Complement Alternat Med 22(4):624-631. https://doi.org/10.1177/2156587217698292

20. Mariappan $V$, Shanthi G (2012) Antimicrobial and phytochemical analysis of Aloe vera L. Int Res J Pharm 3(10):158-161

21. Ranghoo-Sanmukhiya M, Govinden-Soulange J, Lavergne C, Khoyratty S, Da Silva D, Frederich M, Kodja H (2010) Molecular biology, phytochemistry and bioactivity of three endemic Aloe species from Mauritius and Réunion islands. Phytochem Anal 21(6):566-574. https://doi.org/10.1002/pca.1234

22. Kumar S, Yadav A, Yadav M, Yadav JP (2017) Effect of climate change on phytochemical diversity, total phenolic content and in vitro antioxidant activity of Aloe vera (L.) Burm. f. BMC Res Notes 10(1):1-12

23. Arunkumar S, Muthuselvam M (2009) Analysis of phytochemical constituents and antimicrobial activities of Aloe vera $\mathrm{L}$ against clinical pathogens. World J Agric Sci 5(5):572-576

24. Sathyaprabha G, Kumaravel S, Ruffina D, Praveenkumar P (2010) A comparative study on antioxidant, proximate analysis, antimicrobial activity and phytochemical analysis of Aloe vera and Cissus quadrangularis by GCMS. J Pharm Res 3(12):2970-2973

25. Karpagam T, Sugunabai J, Gomathi S, Muhamad N (2019) Phytochemical study in ethanolic leaves extract of Aloe vera using Gas chromatography. Int J Pharm Sci Res 10(2):1470-1473

26. Raphael E (2012) Phytochemical constituents of some leaves extract of Aloe vera and Azadirachta indica plant species. Global Advanced Research Journal of Environmental Science and Toxicology 1(2):014017

27. López A, De Tangil MS, Vega-Orellana O, Ramírez AS, Rico M (2013) Phenolic constituents, antioxidant and preliminary antimycoplasmic activities of leaf skin and flowers of Aloe vera (L.) Burm. f. (syn. A. barbadensis Mill.) from the Canary Islands (Spain). Molecules 18(5):4942-4954. https://doi.org/10.3390/ molecules 18054942 
28. Debnath T, Ghosh M, Lee YM, Nath NC, Lee KG, Lim BO (2018) Identification of phenolic constituents and antioxidant activity of Aloe barbadensis flower extracts. Food Agric Immunol 29(1):27-38

29. Keyhanian S, Stahl-Biskup E (2007) Phenolic constituents in dried flowers of Aloe vera (Aloe barbadensis) and their in vitro antioxidative capacity. Planta Med 73(6):599-602. https://doi.org/10.1055/s-2007-967202

30. Patel DK, Patel K, Dhanabal SP (2012) Phytochemical standardization of Aloe vera extract by HPTLC techniques. J Acute Dis 1(1):47-50

31. Yang QY, Yao CS, Fang WS (2010) A new triglucosylated naphthalene glycoside from Aloe vera L. Fitoterapia 81(1):59-62. https://doi.org/10.1016/j. fitote.2009.07.006

32. Reynolds T (1985) Observations on the phytochemistry of the Aloe leafexudate compounds. Bot J Linn Soc 90:175-199

33. Wu X, Ding W, Zhong J, Wan J, Xie Z (2013) Simultaneous qualitative and quantitative determination of phenolic compounds in Aloe barbadensis Mill by liquid chromatography-mass spectrometry-ion trap-time-of-flight and high-performance liquid chromatography-diode array detector. J Pharm Biomed Anal 80:94-106. https://doi.org/10.1016/j.jpba.2013.02.034

34. Cardarelli M, Rouphael Y, Pellizzoni M, Colla G, Lucini L (2017) Profile of bioactive secondary metabolites and antioxidant capacity of leaf exudates from eighteen Aloe species. Ind Crop Prod 108:44-51. https://doi.org/10.101 6/j.indcrop.2017.06.017

35. Kedarnath NK, Surekha RS, Mahantesh SP, Patil CS (2012) Phytochemical screening and antimicrobial activity of Aloe vera. World Res J Med Aromat Plants 1(1):11-13

36. Bawankar R, Deepti VC, Singh P, Subashkumar R, Vivekanandhan G, Babu S (2013) Evaluation of bioactive potential of an Aloe vera sterol extract. Phytother Res 27(6):864-868. https://doi.org/10.1002/ptr.4827

37. Okamura N, Hine N, Tateyama Y, Nakazawa M, Fujioka T, Mihashi K, Yagi A (1998) Five chromones from Aloe vera leaves. Phytochemistry 49(1):219-223

38. Tanaka M, Misawa E, Ito Y, Habara N, Nomaguchi K, Yamada M, Toida T, Hayasawa $H$, Takase $M$, Inagaki M, Higuchi R (2006) Identification of five phytosterols from Aloe vera gel as anti-diabetic compounds. Biol Pharm Bull 29(7):1418-1422. https://doi.org/10.1248/bpb.29.1418

39. Esua MF, Rauwald JW (2006) Novel bioactive maloyl glucans from Aloe vera gel: isolation, structure elucidation and in vitro bioassays. Carbohydr Res 341(3):355-364. https://doi.org/10.1016/j.carres.2005.11.022

40. Lawrence R, Tripathi P, Jeyakumar E (2009) Isolation, purification and evaluation of antibacterial agents from Aloe vera. Braz J Microbiol 40(4):906915. https://doi.org/10.1590/S1517-83822009000400023

41. Nejatzadeh-Barandozi F (2013) Antibacterial activities and antioxidant capacity of Aloe vera. Org Med Chem Lett 3(1):1-8

42. Vázquez B, Avila G, Segura D, Escalante B (1996) Anti-inflammatory activity of extracts from Aloe vera gel. J Ethnopharmacol 55(1):69-75. https://doi. org/10.1016/S0378-8741(96)01476-6

43. Kammoun M, Miladi S, Ali YB, Damak M, Gargouri Y, Bezzine S (2011) In vitro study of the PLA2 inhibition and antioxidant activities of Aloe vera leaf skin extracts. Lipids Health Dis 10(1):1-7

44. Zhong J, Huang Y, Ding W, Wu X, Wan J, Luo H (2013) Chemical constituents of Aloe barbadensis Miller and their inhibitory effects on phosphodiesterase-4D. Fitoterapia 91:159-165. https://doi.org/10.1016/j. fitote.2013.08.027

45. Rehman NU, Al-Riyami SA, Hussain H, Ali A, Khan AL, Al-Harrasi A (2019) Secondary metabolites from the resins of Aloe vera and Commiphora mukul mitigate lipid peroxidation. Acta Pharma 69(3):433-441. https://doi.org/10.24 78/acph-2019-0027

46. Rehman NU, Hussain H, Khiat M, Al-Riyami SA, Csuk R, Khan HY, Abbas G, Al-Thani GS, Green IR, Al-Harrasi A (2016) Aloeverasides A and B: two bioactive C-Glucosyl chromones from Aloe vera resin. Helv Chim Acta 99(9): 687-690. https://doi.org/10.1002/hlca.201600126

47. Kambizi L, Sultana N, Afolayan AJ (2005) Bioactive compounds isolated from Aloe ferox: A plant traditionally used for the treatment of sexually transmitted infections in the Eastern Cape, South Africa. Pharm Biol 42(8): 636-639. https://doi.org/10.1080/13880200490902581

48. Wintola OA, Afolayan AJ (2011) Phytochemical constituents and antioxidant activities of the whole leaf extract of Aloe ferox Mill. Pharmacogn Mag 7(28): 325-333. https://doi.org/10.4103/0973-1296.90414

49. Fawole OA, Amoo SO, Ndhlala AR, Light ME, Finnie JF, Van Staden J (2010) Anti-inflammatory, anticholinesterase, antioxidant and phytochemical properties of medicinal plants used for pain-related ailments in South Africa. J Ethnopharmacol 127(2):235-241. https://doi.org/10.1016/j.jep.2009.11.015
50. Koyama J, Ogura T, Tagahara K (1994) Naphtho [2, 3-c] furan-4, 9-dione and its derivatives from Aloe ferox. Phytochemistry 37(4):1147-1148. https://doi. org/10.1016/S0031-9422(00)89546-1

51. Celestino VR, Maranhão HM, Vasconcelos CF, Lima CR, Medeiros GC, Araújo AV, Wanderley AG (2013) Acute toxicity and laxative activity of Aloe ferox resin. Rev Bras 23(2):279-283. https://doi.org/10.1590/S0102-695X2013 005000009

52. Magwa ML, Gundidza M, Coopoosamy RM, Mayekiso B (2006) Chemical composition of volatile constituents from the leaves of Aloe ferox. Afr J Biotechnol 5(18):1652-1654

53. Kametani S, Kojima-Yuasa A, Kikuzaki H, Kennedy DO, Honzawa M, MatsuiYuasa I (2007) Chemical constituents of cape aloe and their synergistic growth-inhibiting effect on Ehrlich ascites tumor cells. Biosci Biotechnol Biochem 71(5):1220-1229 https://doi.org/10.1271/bbb.60659

54. Arowosegbe S, Wintola OA, Afolayan AJ (2012) Phytochemical constituents and allelopathic effect of Aloe ferox Mill. root extract on tomato. J Med Plant Res 6(11):2094-2099

55. Loots DT, van der Westhuizen FH, Botes $L$ (2007) Aloe ferox leaf gel phytochemical content, antioxidant capacity, and possible health benefits. J Agric Food Chem 55(17):6891-6896. https://doi.org/10.1021/jf071110t

56. Saritha $V$, Anilakumar KR (2010) Toxicological evaluation of methanol extract of Aloe vera in rats. Int J Pharmaceut Biomed Res 1(5):142-149

57. Tanaka M, Yamada M, Toida T, Iwatsuki K (2012) Safety evaluation of supercritical carbon dioxide extract of Aloe vera gel. J Food Sci 77(1):T2-T9. https://doi.org/10.1111/j.1750-3841.2011.02452.x

58. Sehgal I, Winters WD, Scott M, David A, Gillis G, Stoufflet T, Nair A, Kousoulas K (2013) Toxicologic assessment of a commercial decolorized whole leaf Aloe vera juice, lily of the desert filtered whole leaf juice with aloesorb. J Toxicol 2013:1-12. https://doi.org/10.1155/2013/802453

59. Mwale M, Masika PJ (2012) Toxicological studies on the leaf extract of Aloe ferox Mill. (Aloaceae). Sci Res Essays 7(15):1605-1613

60. Devaraj A, Karpagam T (2011) Evaluation of anti-inflammatory activity and analgesic effect of Aloe vera leaf extract in rats. Int Res J Pharm 2(3):103-110

61. Erhabor JO, Idu M (2017) Aphrodisiac potentials of the ethanol extract of Aloe barbadensis Mill. root in male Wistar rats. BMC Complement Altern Med 17(1):1-10

62. Abosede WO, Sunday JAA (2015) Toxicological investigations of Aloe ferox Mill extracts using Brine shrimp (Artemia salina L.) assay. Pak J Pharm Sci 28(2):635-640

63. Hamidi MR, Jovanova B, Panovska TK (2014) Toxicological evaluation of the plant products using Brine Shrimp (Artemia salina L.) model. Maced Pharm Bull 60(1):9-18

64. Nghonjuyi NW, Tiambo CK, Taïwe GS, Toukala JP, Lisita F, Juliano RS, Kimbi HK (2016) Acute and sub-chronic toxicity studies of three plants used in Cameroonian ethnoveterinary medicine: Aloe vera (L.) Burm. f. (Xanthorrhoeaceae) leaves, Carica papaya L. (Caricaceae) seeds or leaves, and Mimosa pudica L. (Fabaceae) leaves in Kabir chicks. J Ethnopharmacol 178:40-49. https://doi.org/10.1016/j.jep.2015.11.049

65. Shah AH, Qureshi S, Tariq M, Ageel AM (1989) Toxicity studies on six plants used in the traditional Arab system of medicine. Phytother Res 3(1):25-29. https://doi.org/10.1002/ptr.2650030107

66. Ghosh AK, Banerjee M, Mandal TK, Mishra A, Bhowmik MK (2011) A study on analgesic efficacy and adverse effects of Aloe vera in Wistar rats. Pharmacologyonline 1:1098-1108

67. Yimam M, Brownell $L$, Jia Q (2014) In vivo safety evaluation of UP780, a standardized composition of aloe chromone aloesin formulated with an Aloe vera inner leaf fillet. Regul Toxicol Pharmacol 69(3):390-397. https://doi. org/10.1016/j.yrtph.2014.05.001

68. Wintola OA, Sunmonu TO, Afolayan AJ (2011) Toxicological evaluation of aqueous extract of Aloe ferox Mill in loperamide-induced constipated rats. Hum Exp Toxicol 30(5):425-431. https://doi.org/10.1177/096032711 0372647

69. Kwack SJ, Do SG, Kim YW, Kim YJ, Gwak HM, Park HJ, Roh T, Shin MK, Lim SK, Kim HS, Lee BM (2014) The No-Observed-Adverse-Effect Level (NOAEL) of Baby Aloe Powder (BAP) for nutraceutical application based upon toxicological evaluation. J Toxicol Environ Health, Part A 77(22-24):13191331. https://doi.org/10.1080/15287394.2014.951590

70. Koroye OC, Siminialayi IM, Etebu EN (2010) Effects of oral administration of Aloe vera plus on the heart and kidney: a subacute toxicity study in rat models. Nigerian Health Journal 10(1-2):17-21 
71. Sodani IJ (2015) Histopathological changes of male mice kidneys treated with fresh Aloe vera whole leaf extract. Iraqi J Med Sci 13(2):160-166

72. Sudhakar P, Prabhu W, Jamuna B, Adithya RS, Joy A, Anand R (2018) Preclinical toxicological evaluation of Aloe vera health drinks in Wistar rats. Intern J Res Pharm Sci \&Technol 1(1):27-32. https://doi.org/10.33974/ijrpst.v1i1.33

73. Chen T, Wang L, Hu C (2017) Treatment-related changes after short-term exposure of SD rats to Aloe vera whole-leaf freeze-dried powder. Int J Exp Pathol 98(5):248-259. https://doi.org/10.1111/iep.12242

74. Ahbab MA, Korkmaz A, Barlas N, Gürbüz I, Çok I (2014) Biochemical and histological alterations in reproductive tract tissues of male swiss albino mice exposed commercially prepared Aloe vera gel product. Hacettepe J Biol Chem 42:351-360

75. Bala S, Chugh NA, Bansal SC, Garg ML, Koul A (2017) Safety evaluation of Aloe vera pulp aqueous extract based on histoarchitectural and biochemical alterations in mice. Indian J Exp Biol 55:568-575

76. Shao A, Broadmeadow A, Goddard G, Bejar E, Frankos V (2013) Safety of purified decolorized (low anthraquinone) whole leaf Aloe vera $(\mathrm{L})$ Burm. whole leaf juice in a 3-month drinking water toxicity study in F344 rats. Food Chem Toxicol 57:21-31. https://doi.org/10.1016/j.fct.2013.03.002

77. Williams LD, Burdock GA, Shin E, Kim S, Jo TH, Jones KN, Matulka RA (2010) Safety studies conducted on a proprietary high-purity Aloe vera inner leaf fillet preparation, Qmatrix ${ }^{\circledast}$. Regul Toxicol Pharmacol 57(1):90-98. https://doi. org/10.1016/j.yrtph.2010.01.002

78. Boudreau MD, Beland FA, Nichols JA, Pogribna M (2013) Toxicology and carcinogenesis studies of a non-decolorized whole leaf extract of Aloe barbadensis Miller (Aloe vera) in F344/N rats and B6C3F1 mice (drinking water study). Toxicol Sci 577:1-266

79. Akao T, Che Q, Kobashi Q (1996) A purgative action of barbaloin is induced by Eubacterium sp. strain BAR, a human intestinal anaerobe, capable of transforming barbaloin to aloe-emodinanthrone. Biol Pharm Bull 19(1):136138. https://doi.org/10.1248/bpb.19.136

80. Boudreau MD, Olson GR, Tryndyak VP, Bryant MS, Felton RP, Beland FA (2017) From the cover: aloin, a component of the Aloe vera plant leaf, induces pathological changes and modulates the composition of microbiota in the large intestines of F344/N male rats. Toxicol Sci 158(2): 302-318. https://doi.org/10.1093/toxsci/kfx105

81. Quan Y, Gong L, He J, Zhou Y, Liu M, Cao Z, Li Y, Peng C (2019) Aloe emodin induces hepatotoxicity by activating NF-KB inflammatory pathway and P53 apoptosis pathway in zebrafish. Toxicol Lett 306:66-79. https://doi. org/10.1016/j.toxlet.2019.02.007

82. Dong X, Fu J, Yin X, Yang C, Ni J (2017) Aloe-emodin induces apoptosis in human liver $\mathrm{HL}-7702$ cells through Fas death pathway and the mitochondrial pathway by generating reactive oxygen species. Phytother Res 31(6):927-936. https://doi.org/10.1002/ptr.5820

83. Panigrahi GK, Ch R, Mudiam MK, Vashishtha VM, Raisuddin S, Das M (2015) Activity-guided chemo toxic profiling of Cassia occidentalis (CO) seeds: Detection of toxic compounds in body fluids of CO-exposed patients and experimental rats. Chem Res Toxicol 28(6):1120-1132. https://doi.org/10.1 021/acs.chemrestox.5b00056

84. Nesslany F, Simar-Meintières S, Ficheux H, Marzin D (2009) Aloe-emodininduced DNA fragmentation in the mouse in vivo comet assay. Mutat Res Genet Toxicol Environ Mutagen 678(1):13-19. https://doi.org/10.1016/j. mrgentox.2009.06.004

85. Vath P, Wamer WG, Falvey DE (2002) Photochemistry and phototoxicity of aloe emodin. Photochem Photobiol 75(4):346-352. https://doi.org/10.1562/ 0031-8655(2002)0750346PAPOAE2.0.CO2

86. Wu C, Liu Y, Yang Y, Zhang P, Zhong W, Wang Y, Wang Q, Xu Y, Li M, Li X, Zheng M (2020) Analysis of therapeutic targets for SARS-CoV-2 and discovery of potential drugs by computational methods. Acta Pharm Sin B 10(5):766-788. https://doi.org/10.1016/j.apsb.2020.02.008

87. Hoffmann M, Kleine-Weber H, Schroeder S, Krüger N, Herrler T, Erichsen S, Schiergens TS, Herrler G, Wu NH, Nitsche A, Müller MA (2020) SARS-CoV-2 cell entry depends on ACE2 and TMPRSS2 and is blocked by a clinically proven protease inhibitor. Cell 181(2):271-280

88. Adeleye OA, Femi-Oyewo MN, Bamiro OA, Bakre LG, Alabi A, Ashidi JS, Balogun-Agbaje OA, Hassan OM, Fakoya G (2021) Ethnomedicinal herbs in African traditional medicine with potential activity for the prevention, treatment, and management of coronavirus disease 2019. Future J Pharm Sci 7(1):1-4

89. Azer SA (2020) COVID-19: Pathophysiology, diagnosis, complications and Investigational therapeutics. New Microbes New Infect 37:100738 https:// doi.org/10.1016/j.nmni.2020.100738
90. Kumar M, Al Khodor S (2020) Pathophysiology and treatment strategies for COVID-19. J Transl Med 18(1):1-9

91. Ntyonga-Pono MP (2020) COVID-19 infection and oxidative stress: an underexplored approach for prevention and treatment? Pan Afr Med J 35(Suppl 2):12. https://doi.org/10.11604/pamj.2020.35.2.22877

92. Runfeng $L$, Yunlong $H$, Jicheng $H$, Weiqi $P$, Qinhai $M$, Yongxia $S$, Chufang $L$, Jin Z, Zhenhua J, Haiming J, Kui Z (2020) Lianhuaqingwen exerts anti-viral and anti-inflammatory activity against novel coronavirus (SARS-CoV-2). Pharmacol Res 156:104761 https://doi.org/10.1016/j.phrs.2020.104761

93. Rossato L, Negrão FJ, Simionatto S (2020) Could the COVID-19 pandemic aggravate antimicrobial resistance. Am J Infect Control 48(9):1129-1130. https://doi.org/10.1016/j.ajic.2020.06.192 https://doi.org/10.1016/j.ajic.2020. 06.192

94. Mpiana PT, Tshibangu DS, Kilembe JT, Gbolo BZ, Mwanangombo DT, Inkoto CL, Lengbiye EM, Mbadiko CM, Matondo A, Bongo GN, Tshilanda DD (2020) Identification of potential inhibitors of SARS-CoV-2 main protease from Aloe vera compounds: a molecular docking study. Chem Phys Lett 754:137751 https://doi.org/10.1016/j.cplett.2020.137751

95. Vijayalakshmi D, Dhandapani R, Jayaveni S, Jithendra PS, Rose C, Mandal AB (2012) In vitro anti-inflammatory activity of Aloe vera by down regulation of MMP-9 in peripheral blood mononuclear cells. J Ethnopharmacol 141(1): 542-546. https://doi.org/10.1016/j.jep.2012.02.040

96. Egesie UG, Chima KE, Galam NZ (2011) Anti-inflammatory and analgesic effects of aqueous extract of Aloe vera (Aloe barbadensis) in rats. Afr J Biomed Res 14(3):209-212

97. Langmead L, Makins RJ, Rampton DS (2004) Anti-inflammatory effects of Aloe vera gel in human colorectal mucosa in vitro. Aliment Pharmacol Ther 19(5):521-527. https://doi.org/10.1111/j.1365-2036.2004.01874.x

98. Davis RH, Donato JJ, Hartman GM, Haas RC (1994) Anti-inflammatory and wound healing activity of a growth substance in Aloe vera. J Am Podiatr Med Assoc 84(2):77-81. https://doi.org/10.7547/87507315-84-2-77

99. Udupa SL, Udupa AL, Kulkarni DR (1994) Anti-inflammatory and wound healing properties of Aloe vera. Fitoterapia 65(2):141-145

100. Davis RH, Leitner MG, Russo JM, Byrne ME (1989) Anti-inflammatory activity of Aloe vera against a spectrum of irritants. J Am Podiatr Med Assoc 79(6): 263-276. https://doi.org/10.7547/87507315-79-6-263

101. López Z, Femenia A, Núñez-Jinez G, Salazar Zúñiga MN, Cano ME, Espino T, Knauth P (2019) In vitro immunomodulatory effect of food supplement from Aloe vera. Evid Based Complement Alternat Med 2019:1-9 https://doi. org/10.1155/2019/5961742

102. Farahnejad Z, Ghazanfari T, Yaraee R (2011) Immunomodulatory effects of Aloe vera and its fractions on response of macrophages against Candida albicans. Immunopharmacol Immunotoxicol 33(4):676-681. https://doi.org/1 0.3109/08923973.2011.560158

103. Chandu AC, Kumar S, Bhattacharjee C, Debnath S, Kannan KK (2011) Studies on immunomodulatory activity of Aloe vera (Linn). Int J Appl Biol Pharm Technol 2:19-22

104. Madan J, Sharma AK, Inamdar N, Rao HS, Singh R (2008) Immunomodulatory properties of Aloe vera gel in mice. Intern J Green Pharm 2(3):151-154

105. Khan SL, Siddiqui FA (2020) Beta-Sitosterol: As Immunostimulant, Antioxidant and Inhibitor of SARS-CoV-2 Spike Glycoprotein. Arch Pharmacol Ther 2(1):12-16

106. Te Velthuis AJ, van den Worm SH, Sims AC, Baric RS, Snijder EJ, van Hemert MJ (2010) Zn2+ inhibits coronavirus and arterivirus RNA polymerase activity in vitro and zinc ionophores block the replication of these viruses in cell culture. PLoS Pathog 6(11):e1001176 https://doi.org/10.1371/journal.ppat.1001176

107. Abouelela ME, Assaf HK, Abdelhamid RA, Elkhyat ES, Sayed AM, Oszako T, Belbahri L, Zowalaty AE, Abdelkader MS (2021) Identification of potential SARS-CoV-2 main protease and spike protein inhibitors from the Genus Aloe: an in silico study for drug development. Molecules 26(6):1767. https:// doi.org/10.3390/molecules26061767

108. Jeong WY, Kim K (2017) Anti-Propionibacterium acnes and the antiinflammatory effect of Aloe ferox miller components. J Herb Med 9:53-59 https://doi.org/10.1016/j.hermed.2017.03.009

109. Mwale M, Masika PJ (2010) Analgesic and anti-inflammatory activities of Aloe ferox Mill. aqueous extract. Afr J Pharm Pharmacol 4(6):291-297

\section{Publisher's Note}

Springer Nature remains neutral with regard to jurisdictional claims in published maps and institutional affiliations. 